\title{
Convergent synthesis of a galactofuranosylated mannan, the repeating unit of Trichophyton mentagrophytes IFO 5466 and Trichophyton rubrum IFO 5467
}

\author{
Zuchao Ma, Jianjun Zhang and Fanzuo Kong* \\ Research Center for Eco-Environmental Sciences, Academia Sinica, PO Box 2871, Beijing 100085, PR China
}

Received 10 March 2004; accepted 25 March 2004

\begin{abstract}
An undecasaccharide repeating unit of the polysaccharide of Trichophyton mentagrophytes IFO 5466 and Trichophyton rubrum IFO 5467, $\alpha$-D-Man $p-(1 \rightarrow 2)-\alpha$-D-Man $p-(1 \rightarrow 6)-[\beta-D-G a l f-(1 \rightarrow 3)]-\alpha-D-M a n p-(1 \rightarrow 2)-[\beta-D-G a l f-(1 \rightarrow 3)]-\alpha-D-M a n p-$ $(1 \rightarrow 2)-\alpha-\mathrm{D}-\operatorname{Man} p-(1 \rightarrow 2)-\alpha-\mathrm{D}-\operatorname{Man} p-(1 \rightarrow 6)-\alpha-\mathrm{D}-\operatorname{Man} p-(1 \rightarrow 2)-[\beta$-D-Galf-( $1 \rightarrow 3)]-\operatorname{Man} p$ was synthesized as its allyl glycoside by coupling of a octasaccharide trichloroacetimidate donor 19 with a trisaccharide acceptor 28 . The donor 19 and 28 were obtained with allyl 3- $O$-acetyl-2- $O$-benzoyl- $\alpha$-D-mannopyranoside 2 , allyl 3- $O$-acetyl-4,6-di- $O$-benzoyl- $\alpha$-D-mannopyranoside 9, allyl 3,4,6tri- $O$-benzoyl- $\alpha$-D-mannopyranosyl- $(1 \rightarrow 2)$-3,4,6-tri- $O$-benzoyl- $\alpha$-D-mannopyranoside $\quad 13, \quad 6$ - $O$-acetyl-2,3,4-tri- $O$-benzoyl- $\alpha$-Dmannopyranosyl trichloroacetimidate $\mathbf{2 6}$ and 2,3,5,6-tetra- $O$-benzoyl- $\beta$-D-galactofuranosyl trichloroacetimidate $\mathbf{1 6}$ as the key synthons by appropriate combination through simple transformation.

(C) 2004 Elsevier Ltd. All rights reserved.
\end{abstract}

\section{Introduction}

The anthropophilic dermatophytes Trichophyton mentagrophytes and Trichophyton rubrum cause chronic, relatively uninflamed, skin infections of the feet, groin and body. Around $90 \%$ of chronic dermatophyte infections are caused by the fungi $T$. mentagrophytes and T. rubrum. ${ }^{1}$ One of the causes of the chronic infection resides in the immunosuppressive effects of the cell wall components of these organisms. ${ }^{2}$ The cell wall polysaccharides of these fungi are known to be the major immunologically active substances. ${ }^{3}$ The structures of the cell wall polysaccharides of T. mentagrophytes and T. rubrum have been studied and characterized, with two kinds of polysaccharides being found. ${ }^{4}$ One is mannan consisting of an $\alpha-(1 \rightarrow 6)$-linked backbone with $\alpha-(1 \rightarrow 2)$-linked monosaccharide side chains, while the second one is galactomannan consisting of an $\alpha-(1 \rightarrow 2)$ and $\alpha-(1 \rightarrow 6)$-linked mannose backbone with galactofuranose side chains. A possible structure of the galactomannan is shown in Figure 1.

The synthesis of the above galactomannan may be useful for understanding the role galactofuranose plays

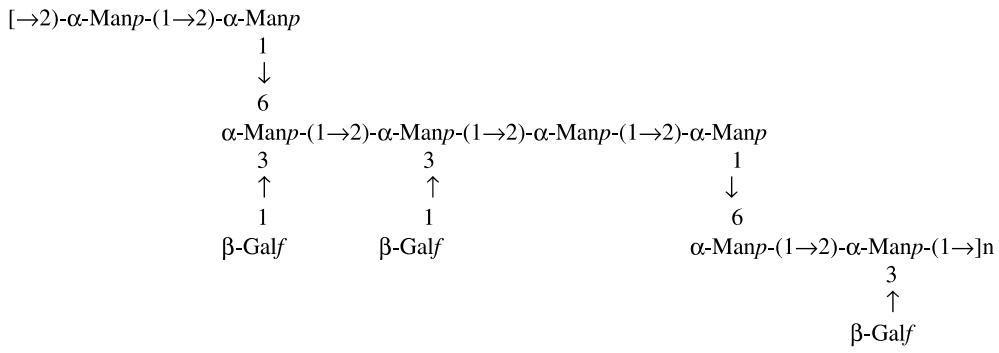

Figure 1. Possible structure of galactomannan of T. mentagrophytes IFO 5466 and T. rubrum IFO 5467.

\footnotetext{
* Corresponding author. Tel.: +86-10-629-366-13; fax: +86-10-629-235-63; e-mail: fzkong@mail.rcees.ac.cn
} 
in microorganisms and for studying the biosynthesis of furanosyl-containing glycoconjugates. The galactomannan could also be used an inhibitor to probe the development of infections or to develop diagnostic methods, or to be used as a vaccine. The synthesis of the above galactomannan is also of interest for synthetic chemists because of its large size and complex structure, which contains different mannose linkages and rare galactofuranose residues. So far there have been very few reports dealing with the synthesis of galactofuranosylated mannan. ${ }^{5}$ We report herein a convergent synthesis of an undecasaccharide, the repeating unit of the polysaccharide of T. mentagrophytes IFO 5466 and T. rubrum IFO 5467.

\section{Results and discussion}

We previously reported a method ${ }^{6}$ for mannose oligosaccharide syntheses using unprotected or lightly protected sugars as the glycosyl acceptors, resulting in a variety of complex oligosaccharides being synthesized efficiently. ${ }^{7}$ In the present research, a concise syntheses of the undecasaccharide repeating unit of the polysaccharide of T. mentagrophytes IFO 5466 and T. rubrum IFO 5467 was achieved. Scheme 1 shows the syntheses of octasaccharides donor 19. Allyl 3-O-acetyl-4,6- $O$ benzylidene- $\alpha$-D-mannopyranoside $\mathbf{1}^{8}$ was chosen as the starting material. Benzoylation of $\mathbf{1}$ followed by debenzylidenation afforded the monosaccharide acceptor 2. Subsequent coupling with perbenzoylated $\alpha-(1 \rightarrow 2)-$ linked mannosyl disaccharide trichloroacetimidate $3^{9}$ selectively produced the $(1 \rightarrow 6)$-linked trisaccharide $4(79 \%)$. The regioselective coupling was confirmed by benzoylation of $\mathbf{4}$ to give $\mathbf{5}$, which showed in its ${ }^{1} \mathrm{H}$ NMR spectrum a new signal at $\delta 5.88 \mathrm{ppm}$ with $J_{3,4}=J_{4,5}=10.1 \mathrm{~Hz}$ for $\mathrm{H}-4$ compared to 4. Deallylation with $\mathrm{PdCl}_{2}{ }^{10}$ followed by trichloroacetimidate formation $^{11}$ yielded trisaccharide donor 7 . The other mannose component containing a potential hydroxyl group at $\mathrm{C}-3$ was prepared from allyl 3-O-acetyl-2- $O$ chloroacetyl- $\alpha$-D-mannopyranoside $\mathbf{8}$. Benzoylation of $\mathbf{8}$ followed by dechloroacetylation with thiourea afforded monosaccharide acceptor 9. Condensation of 9 with donor $\mathbf{7}$ gave tetrasaccharide $\mathbf{1 0}(80 \%)$, and subsequent deallylation followed by trichloroacetimidate formation produced tetrasaccharide donor 12 (73\%), which contained two potential hydroxyl groups at C-3 and C- 3 '. Coupling of donor $\mathbf{1 2}$ with disaccharide acceptor $\mathbf{1 3}^{9}$ afforded hexasaccharide 14 (68\%), whose selective deacetylation ${ }^{12}$ with $\mathrm{MeCOCl} / \mathrm{MeOH}-\mathrm{CH}_{2} \mathrm{Cl}_{2}$ gave hexasaccharide acceptor $15(73 \%)$ with $\mathrm{C}-3^{\prime \prime}$ and $\mathrm{C}-3^{\prime \prime \prime}$ free hydroxyl groups. Coupling of $\mathbf{1 5}$ with 2,3,5,6-tetra- $O$ benzoyl- $\beta$-D-galactofuranosyl trichloroacetimidate $\mathbf{1 6}^{13}$ gave octasaccharide 17 (73\%); subsequent deallylation and trichloroacetimidate formation afforded octasaccharide donor $19(71 \%)$.

Trisaccharide acceptor $\mathbf{2 8}$ was similarly prepared as shown in Scheme 2. Thus, allyl 4,6- $O$-benzylidene- $\alpha$-Dmannopyranoside $\mathbf{2 0}$ was selectively coupled with $\mathbf{1 6}$ to give $\beta$ - $(1 \rightarrow 3)$-linked disaccharide $\mathbf{2 1}$ in good yield $(71 \%)$. The regio- and stereoselectivity were confirmed by acetylation of $\mathbf{2 1}$. The obtained product $\mathbf{2 2}$ showed in its NMR spectrum a sharp singlet at $\delta 5.46$ for $\mathrm{H}-1^{\prime}$ indicating $\beta$-linkage, and a doublet of doublets at 5.42 with $J_{1,2} 1.4 \mathrm{~Hz}$ and $J_{2,3} 3.5 \mathrm{~Hz}$ for $\mathrm{H}-2$ indicating the $3-$ glycosylation. Hydrolysis of $\mathbf{2 2}$ to cleave benzylidene group followed by benzoylation and selective deacetylation produced disaccharide acceptor 25. Condensation of 25 with the donor 6- $O$-acetyl-2,3,4-tri- $O$-benzoyl- $\alpha$-Dmannopyranosyl trichloroacetimidate $\mathbf{2 6}$ gave trisaccharide 27 , and subsequent deacetylation gave the trisaccharide acceptor 28. Finally, coupling of acceptor $\mathbf{2 8}$ with octasaccharide donor 19 afforded the undecasaccharide $29(79 \%)$; debenzoylation in a saturated solution of $\mathrm{NH}_{3}$ in methanol yielded target compound $30(85 \%)$. The ${ }^{1} \mathrm{H}$ and ${ }^{13} \mathrm{C}$ NMR spectra of $\mathbf{3 0}$ showed all of the characteristic signals such as at $\delta 5.20,5.16,5.15,5.11$, 5.07, 5.02, $4.99(7 \mathrm{~s}, 11 \mathrm{H})$ for $\mathrm{H}-1 ; \delta 104.88,104.85$, 104.35 for Gal $f \mathrm{C}-1 ; 102.28,101.71,101.61,100.76$, 100.69, 98.26, 98.10, 97.59 for Man $p$ C-1. A bioassay of sample $\mathbf{3 0}$ is currently in progress, with the results to be reported in due course.

\section{Conclusion}

In summary, a convergent synthesis of a complex galacto-mannosyl undecamer was achieved via a regioand stereoselective manner with readily accessible materials. The described method is suitable for the preparation of other oligosaccharides consisting of mannan backbone linked by either an $\alpha-(1 \rightarrow 2)$ or $\alpha-(1 \rightarrow 6)$ with galactofuranose side chains.

\section{Experimental}

\subsection{General methods}

Optical rotations were determined at $25^{\circ} \mathrm{C}$ with a Perkin-Elmer Model 241-Mc automatic polarimeter. ${ }^{1} \mathrm{H}$ NMR and ${ }^{13} \mathrm{C}$ NMR spectra were recorded with Bruker ARX 400 spectrometers $\left(400 \mathrm{MHz}\right.$ for ${ }^{1} \mathrm{H}, 100 \mathrm{MHz}$ for ${ }^{13} \mathrm{C}$ ) for solutions in $\mathrm{CDCl}_{3}$ or $\mathrm{D}_{2} \mathrm{O}$ as indicated. Chemical shifts are given in ppm downfield from internal $\mathrm{Me}_{4} \mathrm{Si}$. Mass spectra were measured using MALTITOF-MS with CCA as matrix or recorded with a VG PLATFORM mass spectrometer using the ESI mode. Thin-layer chromatography (TLC) was performed on silica gel $\mathrm{HF}_{254}$ with detection by charring with $30 \%$ (v/ v) $\mathrm{H}_{2} \mathrm{SO}_{4}$ in $\mathrm{MeOH}$ or in some cases by a UV detector. Column chromatography was conducted by elution of a column $(16 \times 240 \mathrm{~mm}, 18 \times 300 \mathrm{~mm}, 35 \times 400 \mathrm{~mm})$ of silica gel (100-200 mesh) with EtOAc-petroleum ether $\left(60-90^{\circ} \mathrm{C}\right)$ as the eluent. Solutions were concentrated at $<60^{\circ} \mathrm{C}$ under reduced pressure.

\subsection{General procedure for the glycosylations}

A mixture of the donor and acceptor was dried together under high vacuum for $2 \mathrm{~h}$, then dissolved in anhydrous $\mathrm{CH}_{2} \mathrm{Cl}_{2}$. TMSOTf ( 0.05 equiv) was added dropwise at $-20{ }^{\circ} \mathrm{C}$ with nitrogen protection. The reaction mixture 

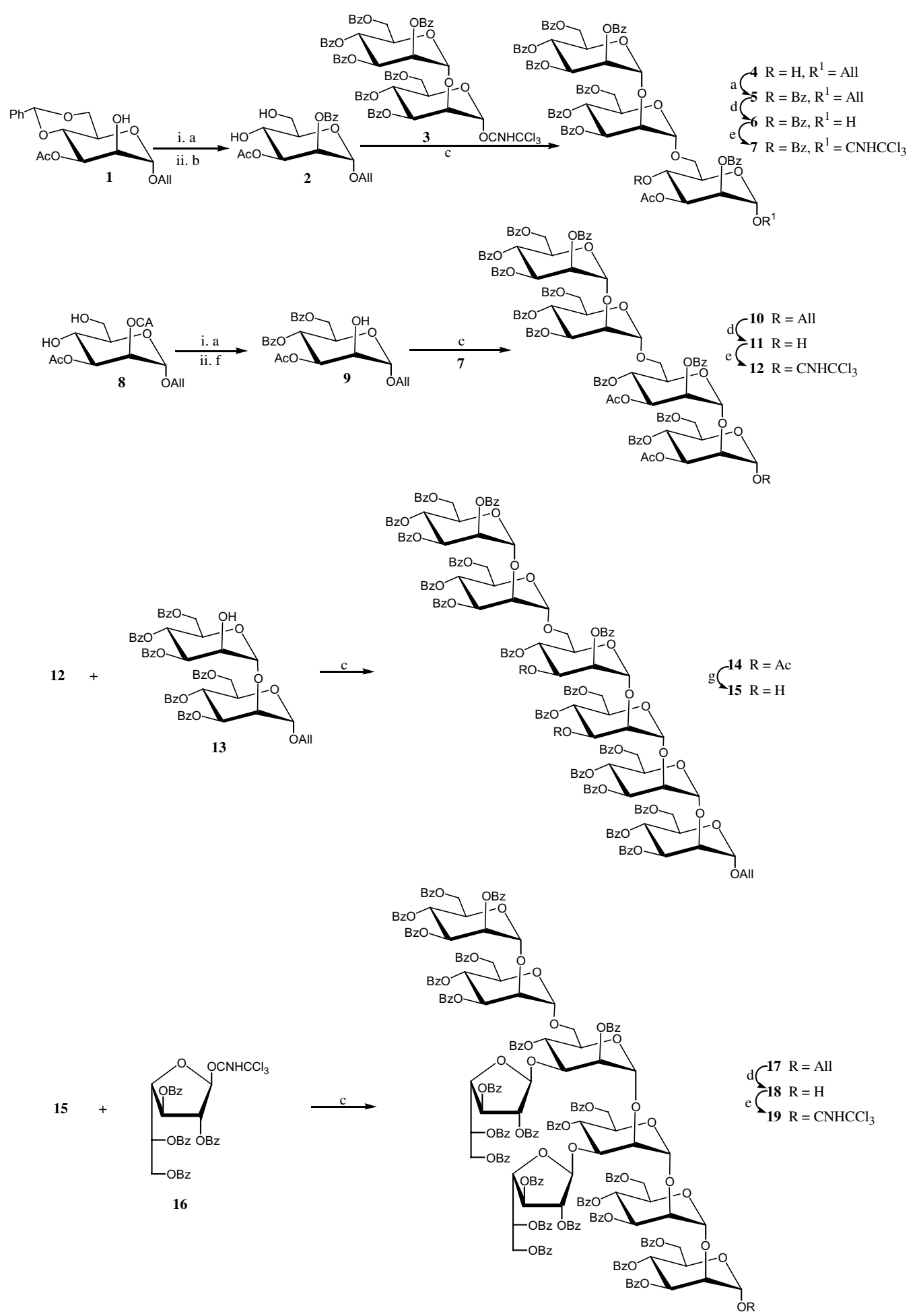

Scheme 1. Reagents and conditions: (a) BzCl-pyridine, $85 \%$ for 5, $88 \%$ for $\mathbf{2 4}$; (b) $90 \%$ TFA, rt, $2 \mathrm{~h}, 87 \%$ for 2, $82 \%$ for $\mathbf{2 3}$; (c) TMSOTf (0.010.05 equiv), $\mathrm{CH}_{2} \mathrm{Cl}_{2},-20$ to $0{ }^{\circ} \mathrm{C}, 2-4 \mathrm{~h}, 79 \%$ for $\mathbf{4}, 80 \%$ for $\mathbf{1 0}, 68 \%$ for $\mathbf{1 4}, 73 \%$ for $\mathbf{1 7}, 71 \%$ for $\mathbf{2 1}, 77 \%$ for 27 and $79 \%$ for 29 , respectively; (d) $\mathrm{PdCl} 2$, $\mathrm{CH}_{3} \mathrm{OH}$, rt, $4 \mathrm{~h}, 81 \%$ for $\mathbf{6}, 81 \%$ for $\mathbf{1 1}, 80 \%$ for $\mathbf{1 8}$; (e) $\mathrm{CCl}_{3} \mathrm{CN}, \mathrm{DBU}, \mathrm{CH}_{2} \mathrm{Cl}_{2}, 2 \mathrm{~h}, 88 \%$ for $7,90 \%$ for $12,89 \%$ for 19 ; (f) $\left(\mathrm{NH}_{2}\right)_{2} \mathrm{CS}_{2} \mathrm{CH}_{2} \mathrm{Cl}_{2}-$ $\mathrm{CH}_{3} \mathrm{OH}$, reflux, 16h, 84\%; (g) methanol/2-6\% $\mathrm{CH}_{3} \mathrm{COCl}$, rt, $12 \mathrm{~h}, 73 \%$ for $\mathbf{1 5}, 75 \%$ for $\mathbf{2 5}, 81 \%$ for $\mathbf{2 8}$; (h) $\mathrm{Ac}_{2} \mathrm{O}-\mathrm{pyridine}, 96 \%$; (i) satd $\mathrm{NH}-\mathrm{MeOH}$, rt, $72 \mathrm{~h}, 85 \%$.

was stirred for $3 \mathrm{~h}$, during which time the temperature was gradually increased to ambient temperature. The mixture was then neutralized with $\mathrm{Et}_{3} \mathrm{~N}$. Concentration of the reaction mixture, followed by purification on a silica-gel column, gave the desired products.

\subsection{Allyl 3-O-acetyl-2- $O$-benzoyl- $\alpha$-D-mannopyranoside} 2

Compound $\mathbf{2}$ was obtained from $\mathbf{1}^{8}$ by benzoylation followed by debenzylidenation. To a solution of $\mathbf{1}$ 


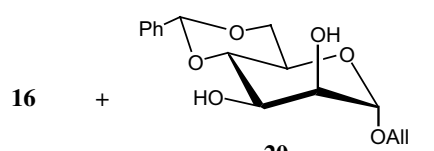

20

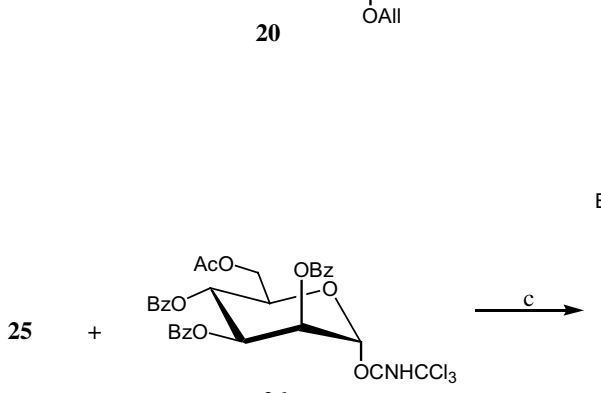

26
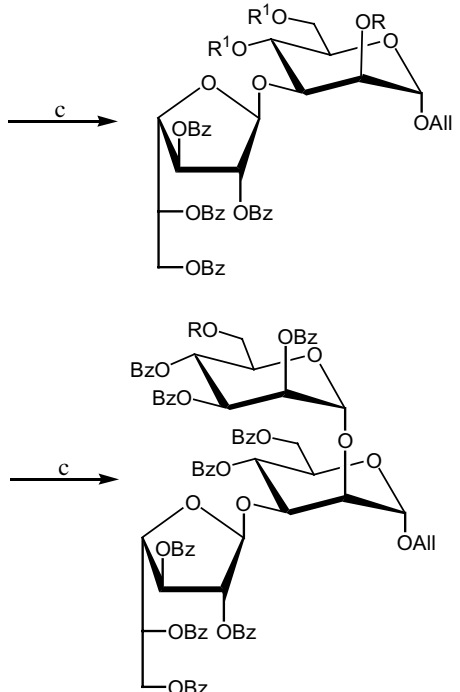

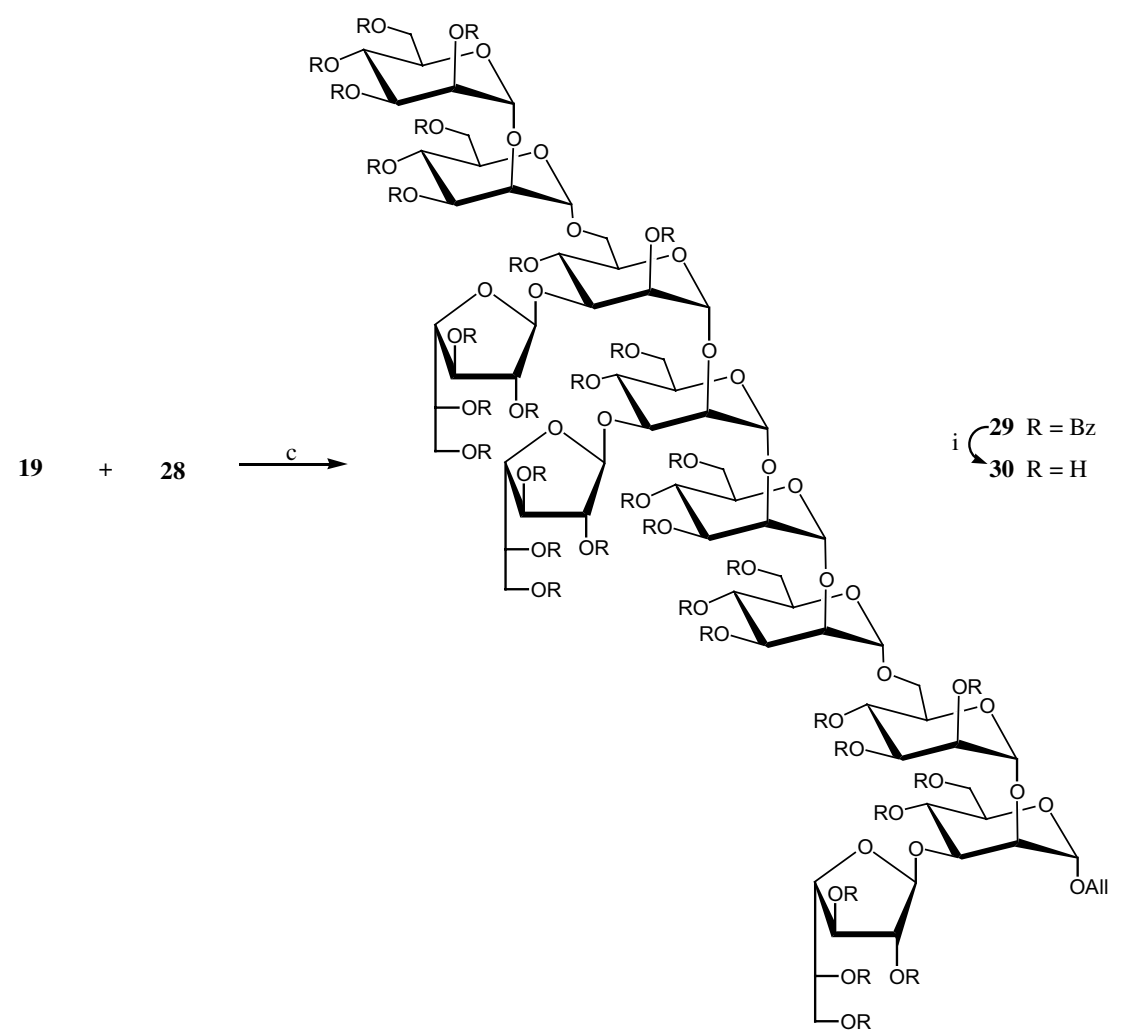

Scheme 2. Reagents and conditions: (a) BzCl-pyridine, $85 \%$ for 5, $88 \%$ for 24; (b) $90 \%$ TFA, rt, 2 h, $87 \%$ for 2, $82 \%$ for 23; (c) TMSOTf (0.010.05 equiv), $\mathrm{CH}_{2} \mathrm{Cl}_{2},-20$ to $0{ }^{\circ} \mathrm{C}, 2-4 \mathrm{~h}, 79 \%$ for $\mathbf{4}, 80 \%$ for $\mathbf{1 0}, 68 \%$ for $\mathbf{1 4}, 73 \%$ for $\mathbf{1 7}, 71 \%$ for $\mathbf{2 1}, 77 \%$ for $\mathbf{2 7}$ and $79 \%$ for $\mathbf{2 9}$, respectively; (d) $\mathrm{PdCl} 2$, $\mathrm{CH}_{3} \mathrm{OH}, \mathrm{rt}, 4 \mathrm{~h}, 81 \%$ for $\mathbf{6}, 81 \%$ for $\mathbf{1 1}, 80 \%$ for $\mathbf{1 8}$; (e) $\mathrm{CCl}_{3} \mathrm{CN}, \mathrm{DBU}, \mathrm{CH}_{2} \mathrm{Cl}_{2}, 2 \mathrm{~h}, 88 \%$ for $7,90 \%$ for $12,89 \%$ for 19 ; (f) $(\mathrm{NH})_{2} \mathrm{CS}, \mathrm{CH}_{2} \mathrm{Cl}_{2}-$ $\mathrm{CH}_{3} \mathrm{OH}$, reflux, $16 \mathrm{~h}, 84 \%$; (g) methanol $/ 2-6 \% \mathrm{CH}_{3} \mathrm{COCl}, \mathrm{rt}, 12 \mathrm{~h}, 73 \%$ for $15,75 \%$ for 25 , $81 \%$ for 28 ; (h) $\mathrm{Ac}{ }_{2} \mathrm{O}-\mathrm{pyridine}$, $96 \%$; (i) satd $\mathrm{NH}-\mathrm{MeOH}$, rt, $72 \mathrm{~h}, 85 \%$.

$(0.70 \mathrm{~g}, 2.0 \mathrm{mmol})$ in pyridine $(5 \mathrm{~mL})$ was added benzoyl chloride $(0.28 \mathrm{~mL}, 2.4 \mathrm{mmol})$. After stirring the mixture overnight at $\mathrm{rt}$, TLC (2:3 petroleum ether-EtOAc) indicated that the reaction was complete. Methanol (2 drops) was added to the reaction mixture and stirring continued for $10 \mathrm{~min}$. Water $(10 \mathrm{~mL})$ was added, the mixture extracted with $\mathrm{CH}_{2} \mathrm{Cl}_{2}(3 \times 10 \mathrm{~mL})$, the extracts were washed with $1 \mathrm{M} \mathrm{HCl}$ and satd aq $\mathrm{NaHCO}_{3}$, dried over $\mathrm{Na}_{2} \mathrm{SO}_{4}$ and concentrated. The residue was dis- solved in $90 \%$ TFA $(10 \mathrm{~mL})$ and stirred for $2 \mathrm{~h}$ at $\mathrm{rt}$, after which TLC (2:1 petroleum ether-EtOAc) indicated that the reaction had gone to completion. The mixture was diluted with toluene $(40 \mathrm{~mL})$ and concentrated in vacuo directly. The residue was passed through a short silica-gel column with 2.5:1 petroleum ether-EtOAc as the eluent to give $2(0.55 \mathrm{~g}, 75 \%$ for two steps) as a syrup. $[\alpha]_{\mathrm{D}}^{25}=-3.5\left(c 1.0, \mathrm{CHCl}_{3}\right) ;{ }^{1} \mathrm{H}$ NMR $(400 \mathrm{MHz}$, $\left.\mathrm{CDCl}_{3}\right): \delta$ 8.06-7.45 (m, 5H, Ph), 5.97-5.97 (m, $1 \mathrm{H}$, 
$\left.-\mathrm{CH}_{2}-\mathrm{CH}=\mathrm{CH}_{2}\right), \quad 5.49 \quad\left(\mathrm{dd}, \quad 1 \mathrm{H}, \quad J_{1,2}=1.7 \mathrm{~Hz}\right.$, $\left.J_{2,3}=3.3 \mathrm{~Hz}, \mathrm{H}-2\right), 5.33\left(\mathrm{dd}, 1 \mathrm{H}, J_{2,3}=3.3 \mathrm{~Hz}, J_{3,4}=\right.$ $10.0 \mathrm{~Hz}, \mathrm{H}-3), 5.35-5.23\left(\mathrm{~m}, 2 \mathrm{H},-\mathrm{CH}_{2}-\mathrm{CH}=\mathrm{CH}_{2}\right), 4.98$ $\left(\mathrm{d}, 1 \mathrm{H}, J_{1,2}=1.7 \mathrm{~Hz}, \mathrm{H}-1\right), 4.26-4.03(\mathrm{~m}, 3 \mathrm{H}, \mathrm{H}-4$, $\left.-\mathrm{CH}_{2}-\mathrm{CH}=\mathrm{CH}_{2}\right), 3.95-3.94(\mathrm{~m}, 2 \mathrm{H}, \mathrm{H}-6), 3.84-3.81$ (m, 1H, H-5), 2.05 (s, 3H, $\left.\mathrm{CH}_{3} \mathrm{CO}\right)$. Anal. Calcd for $\mathrm{C}_{18} \mathrm{H}_{22} \mathrm{O}_{8}$ : C, 59.01; H, 6.05. Found: C, 59.22; H, 6.10.

\subsection{Allyl 2,3,4,6-tetra- $O$-benzoyl- $\alpha$-D-mannopyranosyl- $(1 \rightarrow 2)-3,4,6$-tri- $O$-benzoyl- $\alpha-D-m a n n o p y r a n o s y l-$ $(1 \rightarrow 6)-3-O$-acetyl-2- $O$-benzoyl- $\alpha$-D-mannopyranoside 4}

As described in the general procedure, 2 (0.40 g, $1.1 \mathrm{mmol})$ and $3^{9}(1.1 \mathrm{~g}, 0.92 \mathrm{mmol})$ were coupled, and the product purified by silica-gel column chromatography with 2.5:1 petroleum ether-EtOAc as the eluent to give $4(1.0 \mathrm{~g}, 79 \%)$ as a foamy solid. $[\alpha]_{\mathrm{D}}^{25}=-78.6(c 1.0$, $\left.\mathrm{CHCl}_{3}\right) ;{ }^{1} \mathrm{H}$ NMR $\left(400 \mathrm{MHz}, \mathrm{CDCl}_{3}\right): \delta 8.07-7.23(\mathrm{~m}$, $40 \mathrm{H}, 8 P h), 6.10\left(\mathrm{dd}, 1 \mathrm{H}, J_{3^{\prime \prime} 4^{\prime \prime}}=J_{4^{\prime \prime}} 5^{\prime \prime}=10.0 \mathrm{~Hz}, \mathrm{H}-4^{\prime \prime}\right)$, $6.03\left(\mathrm{dd}, 1 \mathrm{H}, J_{3^{\prime}, 4^{\prime}}=J_{4^{\prime}, 5^{\prime}}=9.8 \mathrm{~Hz}, \mathrm{H}-4^{\prime}\right), 6.06-5.90(\mathrm{~m}$, $\left.3 \mathrm{H}, \mathrm{H}-3^{\prime}, \mathrm{H}-3^{\prime \prime}, \quad-\mathrm{CH}_{2}-\mathrm{CH}=\mathrm{CH}_{2}\right), 5.85(\mathrm{dd}, 1 \mathrm{H}$, $\left.J_{1^{\prime \prime}, 2^{\prime \prime}}=1.8 \mathrm{~Hz}, J_{2^{\prime \prime}, 3^{\prime \prime}}=3.0 \mathrm{~Hz}, \mathrm{H}-2^{\prime \prime}\right), 5.50(\mathrm{dd}, 1 \mathrm{H}$, $\left.J_{1,2}=1.6 \mathrm{~Hz}, J_{2,3}=3.2 \mathrm{~Hz}, \mathrm{H}-2\right), 5.37-5.20(\mathrm{~m}, 4 \mathrm{H}, \mathrm{H}-$ $\left.1^{\prime \prime}, \mathrm{H}-3,-\mathrm{CH}_{2}-\mathrm{CH}=\mathrm{CH}_{2}\right), 5.07\left(\mathrm{~d}, 1 \mathrm{H}, J_{1^{\prime}, 2^{\prime}}=1.5 \mathrm{~Hz}\right.$, $\left.\mathrm{H}-1^{\prime}\right), 4.95\left(\mathrm{~d}, 1 \mathrm{H}, J_{1,2}=1.6 \mathrm{~Hz}, \mathrm{H}-1\right), 4.68-3.85(\mathrm{~m}$, $13 \mathrm{H}, \mathrm{H}-2^{\prime}, \mathrm{H}-4, \mathrm{H}-5, \mathrm{H}-6,-\mathrm{CH}_{2}-\mathrm{CH}=\mathrm{CH}_{2}$ ), 2.07 (s, $3 \mathrm{H}, \mathrm{CH}_{3} \mathrm{CO}$ ) Anal. Calcd for $\mathrm{C}_{79} \mathrm{H}_{70} \mathrm{O}_{25}: \mathrm{C}, 66.85 ; \mathrm{H}$, 4.97. Found: C, 67.09; H, 5.03.

\subsection{Allyl 2,3,4,6-tetra- $O$-benzoyl- $\alpha$-D-mannopyranosyl- $(1 \rightarrow 2)-3,4,6$-tri- $O$-benzoyl- $\alpha$-D-mannopyranosyl- $(1 \rightarrow 6)-3-O$-acetyl-2,4-di- $O$-benzoyl- $\alpha$-D-mannopyrano- side 5}

To a solution of $\mathbf{4}(0.98 \mathrm{~g}, 0.69 \mathrm{mmol})$ in pyridine $(10 \mathrm{~mL})$ was added benzoyl chloride $(97 \mu \mathrm{L}, 0.83 \mathrm{mmol})$. After stirring the mixture overnight at $\mathrm{rt}$, TLC (2:1 petroleum ether-EtOAc) indicated that the reaction was complete. Methanol (2 drops) was added to the reaction mixture, and stirring was continued for $10 \mathrm{~min}$. Water $(20 \mathrm{~mL})$ was added, the mixture extracted with $\mathrm{CH}_{2} \mathrm{Cl}_{2}(3 \times 20 \mathrm{~mL})$, the extract washed with $1 \mathrm{M} \mathrm{HCl}$ and satd aq $\mathrm{NaHCO}_{3}$, dried over $\mathrm{Na}_{2} \mathrm{SO}_{4}$ and concentrated. Purification by flash chromatography (2.5:1 petroleum ether-EtOAc) gave $\mathbf{5}$ as a foamy solid $(0.90 \mathrm{~g}, 85 \%) .[\alpha]_{\mathrm{D}}^{25}=-70.2(c \quad 1.0$, $\left.\mathrm{CHCl}_{3}\right) ;{ }^{1} \mathrm{H}$ NMR $\left(400 \mathrm{MHz}, \mathrm{CDCl}_{3}\right): \delta 8.04-7.26(\mathrm{~m}$, $45 \mathrm{H}, 9 P h), 6.10\left(\mathrm{dd}, 1 \mathrm{H}, J_{3^{\prime \prime}} 4^{\prime \prime}=J_{4^{\prime \prime}} 5^{\prime \prime}=10.1 \mathrm{~Hz}, \mathrm{H}-4^{\prime \prime}\right)$, 6.06-5.96 (m, 4H, H-3', H-3", H-4 $\left.{ }^{\prime},-\mathrm{CH}_{2}-\mathrm{CH}=\mathrm{CH}_{2}\right)$, $5.88\left(\mathrm{dd}, 1 \mathrm{H}, J_{3,4}=J_{4,5}=10.1 \mathrm{~Hz}, \mathrm{H}-4\right), 5.84(\mathrm{dd}, 1 \mathrm{H}$, $\left.J_{1^{\prime \prime}, 2^{\prime \prime}}=1.1 \mathrm{~Hz}, J_{2^{\prime \prime}, 3^{\prime \prime}}=3.0 \mathrm{~Hz}, \mathrm{H}-2^{\prime \prime}\right), 5.74$ (dd, $1 \mathrm{H}$, $\left.J_{2,3}=3.3 \mathrm{~Hz}, \quad J_{3,4}=10.1 \mathrm{~Hz}, \quad \mathrm{H}-3\right), \quad 5.60 \quad(\mathrm{dd}, \quad 1 \mathrm{H}$, $\left.J_{1.2}=1.4 \mathrm{~Hz}, J_{2,3}=3.3 \mathrm{~Hz}, \mathrm{H}-2\right), 5.46-5.29(\mathrm{~m}, 2 \mathrm{H}$, $\left.-\mathrm{CH}_{2}-\mathrm{CH}=\mathrm{CH}_{2}\right), 5.23\left(\mathrm{~d}, 1 \mathrm{H}, J_{1^{\prime \prime}} .^{\prime \prime}=1.1 \mathrm{~Hz}, \mathrm{H}-1^{\prime \prime}\right), 5.10$ $\left(\mathrm{d}, 1 \mathrm{H}, J_{1^{\prime}, 2^{\prime}}=1.3 \mathrm{~Hz}, \mathrm{H}-1^{\prime}\right), 5.08\left(\mathrm{~d}, 1 \mathrm{H}, J_{1,2}=1.4 \mathrm{~Hz}, \mathrm{H}-\right.$ 1), 4.65-3.66 (m, 12H, H-2', H-5, H-6, $-\mathrm{CH}_{2}-\mathrm{CH}=\mathrm{CH}_{2}$ ), 1.89 (s, 3H, CH $\left.\mathrm{CH}_{3} \mathrm{CO}\right) ;{ }^{13} \mathrm{C} \mathrm{NMR}\left(100 \mathrm{MHz}, \mathrm{CDCl}_{3}\right): \delta$ $170.01,166.10,165.91,165.60,165.46,165.40,165.38$, $165.22,164.95,164.78,118.38,99.79,98.46,96.73,70.54$ 70.39, 70.06, 69.74, 69.62, 69.55, 69.33, 68.86, 68.77, 67.22, 67.13, 66.62, 66.33, 63.46, 62.62, 20.60. Anal. Calcd for $\mathrm{C}_{86} \mathrm{H}_{74} \mathrm{O}_{26}$ : C, 67.80; H, 4.90. Found: C, 67.66; H, 4.97.
4.6. 2,3,4,6-Tetra- $O$-benzoyl- $\alpha$-D-mannopyranosyl$(1 \rightarrow 2)-3,4,6$-tri- $O$-benzoyl- $\alpha$-D-mannopyranosyl$(1 \rightarrow 6)-3-O$-acetyl-2,4-di- $O$-benzoyl- $\alpha$-D-mannopyranosyl trichloroacetimidate 7

To a solution of $5(0.89 \mathrm{~g}, 0.58 \mathrm{mmol})$ in anhydrous $\mathrm{MeOH}(10 \mathrm{~mL})$ was added $\mathrm{PdCl}_{2}(30 \mathrm{mg})$. After stirring the mixture at $\mathrm{rt}$ for $2 \mathrm{~h}$, TLC $(2: 1$ petroleum etherEtOAc) indicated that the reaction was complete. The mixture was filtered, the solution concentrated to dryness and the resultant residue purified by flash chromatography $(2.5: 1$ petroleum ether-EtOAc) to give $6(0.70 \mathrm{~g}$, $81 \%)$ as a white foam. A mixture of $6(0.70 \mathrm{~g}, 0.47 \mathrm{mmol})$, trichloroacetonitrile $(94 \mu \mathrm{L}, 0.94 \mathrm{mmol})$ and 1,8-diazabicyclo[5.4.0]-undecene (DBU) $(30 \mu \mathrm{L})$ in dry $\mathrm{CH}_{2} \mathrm{Cl}_{2}$ $(10 \mathrm{~mL})$ was stirred under nitrogen for $3 \mathrm{~h}$ and then concentrated. The residue was purified by flash chromatography (3:1 petroleum ether-EtOAc) to give $7(0.67 \mathrm{~g}$, $88 \%)$ as a foamy solid: $[\alpha]_{\mathrm{D}}^{25}=-68.4\left(\mathrm{c} 1.0, \mathrm{CHCl}_{3}\right) ;{ }^{1} \mathrm{H}$ NMR $\left(400 \mathrm{MHz}, \mathrm{CDCl}_{3}\right): \delta 9.01\left(\mathrm{~s}, 1 \mathrm{H}, \mathrm{CNHCCl} \mathrm{Cl}_{3}\right.$ ), 8.15-7.26 (m, 45H, 9Ph), 6.51 (s, 1H, H-1), $6.10(\mathrm{dd}, 1 \mathrm{H}$, $\left.J_{3^{\prime \prime} .4^{\prime \prime}}=J_{4^{\prime \prime}}, 5^{\prime \prime}=10.1 \mathrm{~Hz}, \mathrm{H}-4^{\prime \prime}\right), 6.05-5.80(\mathrm{~m}, 7 \mathrm{H}, \mathrm{H}-2$, H-2", H-3, H-3', H-3", H-4, H-4'), 5.22 (s, 1H, H-1"), 5.12 (s, 1H, H-1'), 4.66-3.69 (m, 10H, H-2', H-5, H-6), 1.92 (s, $3 \mathrm{H}, \mathrm{CH}_{3} \mathrm{CO}$ ). Anal. Calcd for $\mathrm{C}_{85} \mathrm{H}_{70} \mathrm{Cl}_{3} \mathrm{NO}_{26}$ : C, 62.72; H, 4.33. Found: C, 62.82; H, 4.41.

\subsection{Allyl 3-O-acetyl-4,6-di- $O$-benzoyl- $\alpha$-D-mannopyr- anoside 9}

Compound 8 (0.34 g, $1.0 \mathrm{mmol})$ was benzoylated under the same conditions as those used in the preparation of 5 from $\mathbf{4}$, to give a residue. To a solution of the residue in $\mathrm{MeOH}(15 \mathrm{~mL})-\mathrm{CH}_{2} \mathrm{Cl}_{2}(20 \mathrm{~mL})$ was added thiourea $(0.15 \mathrm{~g})$, and the mixture was refluxed for $16 \mathrm{~h}$, after which TLC (1:1 petroleum ether-EtOAc) indicated that the reaction had gone to completion. The mixture was then concentrated and the residue passed through a silica-gel column with 3:1 petroleum ether-EtOAc as the eluent to give $9(0.33 \mathrm{~g}, 71 \%$ for two steps $)$ as a foamy solid. $[\alpha]_{\mathrm{D}}^{25}=+43.6\left(c 1.0, \mathrm{CHCl}_{3}\right) ;{ }^{1} \mathrm{H} \mathrm{NMR}(400 \mathrm{MHz}$, $\left.\mathrm{CDCl}_{3}\right): \delta 8.03-7.37(\mathrm{~m}, 10 \mathrm{H}, 2 \mathrm{Ph}), 5.98-5.89(\mathrm{~m}, 1 \mathrm{H}$, $\left.-\mathrm{CH}_{2}-\mathrm{CH}=\mathrm{CH}_{2}\right), 5.73\left(\mathrm{dd}, 1 \mathrm{H}, J_{3,4}=J_{4,5}=10.0 \mathrm{~Hz}, \mathrm{H}-\right.$ 4), $5.56\left(\mathrm{dd}, 1 \mathrm{H}, J_{2,3}=3.2 \mathrm{~Hz}, J_{3,4}=10.0 \mathrm{~Hz}, \mathrm{H}-3\right)$, 5.34-5.22 (m, 2H, $\left.-\mathrm{CH}_{2}-\mathrm{CH}=\mathrm{CH}_{2}\right), 5.00(\mathrm{~d}, 1 \mathrm{H}$, $\left.J_{1,2}=1.6 \mathrm{~Hz}, \quad \mathrm{H}-1\right), \quad 4.55 \quad\left(\mathrm{dd}, 1 \mathrm{H}, \quad J_{5,6 \mathrm{a}}=5.1 \mathrm{~Hz}\right.$, $\left.J_{6 \mathrm{a}, 6 \mathrm{~b}}=12.0 \mathrm{~Hz}, \mathrm{H}-6 \mathrm{a}\right), 4.44\left(\mathrm{dd}, 1 \mathrm{H}, J_{5,6 \mathrm{~b}}=5.5 \mathrm{~Hz}\right.$, $\left.J_{6 \mathrm{a}, 6 \mathrm{~b}}=12.0 \mathrm{~Hz}, \mathrm{H}-6 \mathrm{~b}\right), 4.32-4.06$ (m, 4H, H-2, H-5, $\left.-\mathrm{CH}_{2}-\mathrm{CH}=\mathrm{CH}_{2}\right), 2.00\left(\mathrm{~s}, 3 \mathrm{H}, \mathrm{CH}_{3} \mathrm{CO}\right)$. Anal. Calcd for $\mathrm{C}_{25} \mathrm{H}_{26} \mathrm{O}_{9}$ : C, 63.82; H, 5.57. Found: C, 63.96; H, 5.62.

\subsection{Allyl 2,3,4,6-tetra- $O$-benzoyl- $\alpha$-D-mannopyranosyl- $(1 \rightarrow 2)-3,4,6$-tri- $O$-benzoyl- $\alpha$-D-mannopyranosyl- $(1 \rightarrow 6)-3-O$-acetyl-2,4-di- $O$-benzoyl- $\alpha$-D-mannopyrano- syl-( $1 \rightarrow 2)$-3- $O$-acetyl-4,6-di- $O$-benzoyl- $\alpha$-D-mannopyr- anoside 10}

Donor $7(0.65 \mathrm{~g}, 0.40 \mathrm{mmol})$ was coupled with acceptor $9(0.22 \mathrm{~g}, 0.48 \mathrm{mmol})$ as described in the general procedure, and the product purified by chromatography with 2:1 petroleum ether-EtOAc as the eluent to give $\mathbf{1 0}$ 
$(0.62 \mathrm{~g}, 80 \%)$ as a foamy solid. $[\alpha]_{\mathrm{D}}^{25}=-71.2$ (c 1.0, $\left.\mathrm{CHCl}_{3}\right) ;{ }^{1} \mathrm{H} \mathrm{NMR}\left(400 \mathrm{MHz}, \mathrm{CDCl}_{3}\right): \delta 8.06-7.26(\mathrm{~m}$, $55 \mathrm{H}, 11 P h), 6.11\left(\mathrm{dd}, 1 \mathrm{H}, J_{3^{\prime \prime \prime} 4^{\prime \prime \prime}}=J_{4^{\prime \prime \prime}} 5^{\prime \prime \prime}=10.0 \mathrm{~Hz}, \mathrm{H}-\right.$ $\left.4^{\prime \prime \prime}\right), 6.10\left(\mathrm{dd}, 1 \mathrm{H}, J_{3^{\prime \prime}, 4^{\prime \prime}}=J_{4^{\prime \prime}} .^{\prime \prime}=10.0 \mathrm{~Hz}, \mathrm{H}-4^{\prime \prime}\right), 6.04$ 5.75 (m, 7H, H-2' ${ }^{\prime \prime \prime}, \mathrm{H}_{-3}{ }^{\prime}, \mathrm{H}-3^{\prime \prime}, \mathrm{H}-3^{\prime \prime \prime}, \mathrm{H}-4, \mathrm{H}-4^{\prime},-\mathrm{CH}_{2}-$ $\left.\mathrm{CH}=\mathrm{CH}_{2}\right), 5.70\left(\mathrm{dd}, 1 \mathrm{H}, J_{1^{\prime}, 2^{\prime}}=1.7 \mathrm{~Hz}, J_{2^{\prime}, 3^{\prime}}=3.1 \mathrm{~Hz}\right.$, $\left.\mathrm{H}-2^{\prime}\right), 5.67\left(\mathrm{dd}, 1 \mathrm{H}, J_{2,3}=3.2 \mathrm{~Hz}, J_{3.4}=9.8 \mathrm{~Hz}, \mathrm{H}-3\right)$, $5.28\left(\mathrm{~d}, 1 \mathrm{H}, J_{1^{\prime \prime \prime}} .^{\prime \prime \prime}=1.6 \mathrm{~Hz}, \mathrm{H}-1^{\prime \prime \prime}\right), 5.26-5.12(\mathrm{~m}, 2 \mathrm{H}$, $\left.-\mathrm{CH}_{2}-\mathrm{CH}=\mathrm{CH}_{2}\right), 5.24\left(\mathrm{~d}, 1 \mathrm{H}, J_{1^{\prime \prime}} 2^{\prime \prime}=1.5 \mathrm{~Hz}, \mathrm{H}-1^{\prime \prime}\right)$, $5.10\left(\mathrm{~d}, \quad 1 \mathrm{H}, \quad J_{1^{\prime}, 2^{\prime}}=1.7 \mathrm{~Hz}, \mathrm{H}-1^{\prime}\right), 4.98(\mathrm{~d}, 1 \mathrm{H}$, $\left.J_{1,2}=1.9 \mathrm{~Hz}, \mathrm{H}-1\right), 4.63-3.64$ (m, 16H, H-2', H-2" , H-5, $\left.\mathrm{H}-6,-\mathrm{CH}_{2}-\mathrm{CH}=\mathrm{CH}_{2}\right), 2.06\left(\mathrm{~s}, 3 \mathrm{H}, \mathrm{CH}_{3} \mathrm{CO}\right), 1.94$ (s, $\left.3 \mathrm{H}, \mathrm{CH}_{3} \mathrm{CO}\right) ;{ }^{13} \mathrm{C} \mathrm{NMR}\left(100 \mathrm{MHz}, \mathrm{CDCl}_{3}\right): \delta 170.45$, $169.84,166.29,166.17,165.95,165.50,165.47,165.43$, $165.38,164.81,163.38,99.89,99.83,98.93,97.95,70.73$, $70.43,70.28,70.08,69.87,69.60,69.05,68.96,68.93$, $68.73,67.91,67.14,66.64,66.56,65.91,63.77,63.38$, $62.50,60.38,21.02,20.72$. Anal. Calcd for $\mathrm{C}_{108} \mathrm{H}_{94} \mathrm{O}_{34}$ : C, 67.01; H, 4.89. Found: C, 66.88; H, 4.79.

\subsection{2,3,4,6-Tetra- $O$-benzoyl- $\alpha$-D-mannopyranosyl- $(1 \rightarrow 2)-3,4,6$-tri- $O$-benzoyl- $\alpha$-D-mannopyranosyl- $(1 \rightarrow 6)-3-O$-acetyl-2,4-di- $O$-benzoyl- $\alpha$-D-mannopyranos- yl-(1 $\rightarrow$ )-3- $O$-acetyl-4,6-di- $O$-benzoyl- $\alpha$-D-mannopyr- anosyl trichloroacetimidate 12}

Deallylation of tetrasaccharide $10(0.60 \mathrm{~g}, 0.31 \mathrm{mmol})$ followed by trichloroacetimidation under the same conditions as those used in the preparation of $\mathbf{7}$ from $\mathbf{5}$ gave a residue, which was purified by flash chromatography (2:1 petroleum ether-EtOAc) to give $12(0.46 \mathrm{~g}$, $73 \%$ for two steps) as a foamy solid. $[\alpha]_{\mathrm{D}}^{25}=-69.5$ (c 1.0, $\left.\mathrm{CHCl}_{3}\right) ;{ }^{1} \mathrm{H}$ NMR $\left(400 \mathrm{MHz}, \mathrm{CDCl}_{3}\right): \delta 8.88(\mathrm{~s}, 1 \mathrm{H}$, $\left.\mathrm{CNHCCl}_{3}\right), 8.05-7.26(\mathrm{~m}, 55 \mathrm{H}, 11 \mathrm{Ph}), 6.56(\mathrm{~s}, 1 \mathrm{H}, \mathrm{H}-1)$, $6.12\left(\mathrm{dd}, 1 \mathrm{H}, J_{3^{\prime \prime \prime}} 4^{\prime \prime \prime}=J_{4^{\prime \prime \prime}} 5^{\prime \prime \prime}=10.0 \mathrm{~Hz}, \mathrm{H}-4^{\prime \prime \prime}\right), 6.09(\mathrm{dd}$, $\left.1 \mathrm{H}, J_{3^{\prime \prime} 4^{\prime \prime}}=J_{4^{\prime \prime} 5^{\prime \prime}}=10.0 \mathrm{~Hz}, \mathrm{H}-4^{\prime \prime}\right), 6.04-5.68(\mathrm{~m}, 8 \mathrm{H}$, H-2', H-2'"', H-3, H-3', H-3", H-3"', H-4, H-4'), 5.36 (s, $\left.1 \mathrm{H}, \mathrm{H}-1^{\prime \prime \prime}\right), \quad 5.25 \quad\left(\mathrm{~s}, 1 \mathrm{H}, \mathrm{H}-1^{\prime \prime}\right), \quad 5.00 \quad(\mathrm{~d}, 1 \mathrm{H}$, $\left.J_{1^{\prime}, 2^{\prime}}=1.7 \mathrm{~Hz}, \mathrm{H}-1^{\prime}\right)$, 4.67-3.72 (m, 14H, H-2', H-2", $\mathrm{H}-$ 5, H-6), 2.09 (s, 3H, CH $\left.\mathrm{CH}_{3} \mathrm{CO}\right), 1.95$ (s, 3H, $\mathrm{CH}_{3} \mathrm{CO}$ ). Anal. Calcd for $\mathrm{C}_{107} \mathrm{H}_{90} \mathrm{Cl}_{3} \mathrm{NO}_{34}$ : C, 62.99; $\mathrm{H}, 4.45$. Found: C, 63.22; H, 4.53.

4.10. Allyl 2,3,4,6-tetra- $O$-benzoyl- $\alpha$-D-mannopyranosyl$(1 \rightarrow 2)-3,4,6$-tri- $O$-benzoyl- $\alpha$-D-mannopyranosyl$(1 \rightarrow 6)-3-O$-acetyl-2,4-di- $O$-benzoyl- $\alpha$-D-mannopyranosyl-(1 $\rightarrow$ 2)-3- $O$-acetyl-4,6-di- $O$-benzoyl- $\alpha$-D-mannopyranosyl-( $1 \rightarrow 2)-3,4,6$-tri- $O$-benzoyl- $\alpha$-D-mannopyranosyl$(1 \rightarrow 2)-3,4,6$-tri- $O$-benzoyl- $\alpha$-D-mannopyranoside 14

Compound 12 (0.46g, $0.22 \mathrm{mmol})$ and $13(0.27 \mathrm{~g}$, $0.27 \mathrm{mmol}$ ) were coupled under the same conditions as those used in the preparation of $\mathbf{1 0}$ from $\mathbf{7}$ and $\mathbf{9}$, giving $17(0.44 \mathrm{~g}, 68 \%)$ as a foamy solid. $[\alpha]_{\mathrm{D}}^{25}=-16.5(\mathrm{c} 1.0$, $\left.\mathrm{CHCl}_{3}\right) ;{ }^{1} \mathrm{H}$ NMR $\left(400 \mathrm{MHz}, \mathrm{CDCl}_{3}\right): \delta 8.13-7.26(\mathrm{~m}$, $85 \mathrm{H}, 17 P h), 6.26\left(\mathrm{dd}, 1 \mathrm{H}, J_{3,4}=J_{4.5}=10.0 \mathrm{~Hz}, \mathrm{H}-4\right)$, 6.14-5.65 (m, 14H, 2H-2, 6H-3, 5H-4, $-\mathrm{CH}_{2}-$ $\left.\mathrm{CH}=\mathrm{CH}_{2}\right), 5.42\left(\mathrm{~d}, 1 \mathrm{H}, J_{1,2}=1.6 \mathrm{~Hz}, \mathrm{H}-1\right), 5.30-5.18$ $\left(\mathrm{m}, 2 \mathrm{H},-\mathrm{CH}_{2}-\mathrm{CH}=\mathrm{CH}_{2}\right), 5.27\left(\mathrm{~d}, 1 \mathrm{H}, J_{1,2}=1.2 \mathrm{~Hz}, \mathrm{H}-\right.$ 1), $5.22\left(\mathrm{~d}, 1 \mathrm{H}, J_{1,2}=1.1 \mathrm{~Hz}, \mathrm{H}-1\right), 5.11(\mathrm{~d}, 1 \mathrm{H}$, $\left.J_{1,2}=1.4 \mathrm{~Hz}, \mathrm{H}-1\right), 5.08$ (d, 1H, $\left.J_{1,2}=1.1 \mathrm{~Hz}, \mathrm{H}-1\right), 4.94$ $\left(\mathrm{d}, 1 \mathrm{H}, J_{1,2}=1.3 \mathrm{~Hz}, \mathrm{H}-1\right), 4.67-3.72(\mathrm{~m}, 24 \mathrm{H}, 4 \mathrm{H}-2$, $\left.6 \mathrm{H}-5,12 \mathrm{H}-6,-\mathrm{CH}_{2}-\mathrm{CH}=\mathrm{CH}_{2}\right), 2.04$ (s, 3H, $\mathrm{CH}_{3} \mathrm{CO}$ ), 1.97 (s, 3H, CH$\left.H_{3} \mathrm{CO}\right) ;{ }^{13} \mathrm{C} \mathrm{NMR}\left(100 \mathrm{MHz}, \mathrm{CDCl}_{3}\right): \delta$ $170.05,169.89,166.14,166.04,165.99,165.66,165.61$, $165.58,165.57,165.50,165.43,165.38,165.32,165.30$, $164.81,163.38,100.26,100.13,99.76,99.64,98.84$, $97.99,71.09,70.38,70.26,70.11,69.96,69.86,69.70$, $69.58,68.99,68.85,68.75,67.89,67.45,67.12,67.04$, $66.33,66.10,65.19,63.68,63.57,63.23,61.98,60.34$, 20.76, 20.65. Anal. Calcd for $\mathrm{C}_{162} \mathrm{H}_{138} \mathrm{O}_{50}: \mathrm{C}, 67.45 ; \mathrm{H}$, 4.82. Found: C, 67.68; H, 4.73.

4.11. Allyl 2,3,4,6-tetra- $O$-benzoyl- $\alpha$-D-mannopyranosyl$(1 \rightarrow 2)-3,4,6$-tri- $O$-benzoyl- $\alpha$-D-mannopyranosyl$(1 \rightarrow 6)-2,4-d i-O$-benzoyl- $\alpha-D-m a n n o p y r a n o s y l-(1 \rightarrow 2)-$ 4,6-di- $O$-benzoyl- $\alpha$-D-mannopyranosyl- $(1 \rightarrow 2)-3,4,6$-tri$O$-benzoyl- $\alpha$-D-mannopyranosyl- $(1 \rightarrow 2)-3,4,6$-tri- $O$-benzoyl- $\alpha$-D-mannopyranoside 15

To a solution of $14(0.42 \mathrm{~g}, 0.15 \mathrm{mmol})$ in anhydrous $\mathrm{CH}_{2} \mathrm{Cl}_{2}(5 \mathrm{~mL})$ was added anhydrous $\mathrm{MeOH}(25 \mathrm{~mL})$, then acetyl chloride $(1 \mathrm{~mL})$ added to the reaction mixture at $0^{\circ} \mathrm{C}$. The solution was stoppered in a flask and stirred at rt until TLC (1:1 petroleum ether-EtOAc) showed that the reaction was complete. The solution was neutralized with $\mathrm{Et}_{3} \mathrm{~N}$, then concentrated to dryness. The residue was passed through a short silica-gel column to give $15(0.30 \mathrm{~g}, 73 \%)$ as a foamy solid. $\left.[\alpha]_{\mathrm{D}}^{25}=+12.7(c) 1.0, \mathrm{CHCl}_{3}\right) ; \delta 8.04-7.15(\mathrm{~m}, 85 \mathrm{H}$, $17 P h), 6.12\left(\mathrm{dd}, 1 \mathrm{H}, J_{3,4}=J_{4.5}=10.0 \mathrm{~Hz}, \mathrm{H}-4\right), 6.08-$ $5.51\left(\mathrm{~m}, 12 \mathrm{H}, 2 \mathrm{H}-2,4 \mathrm{H}-3,5 \mathrm{H}-4,-\mathrm{CH}_{2}-\mathrm{CH}=\mathrm{CH}_{2}\right), 5.36$ (s, 1H, H-1), 5.31 (s, 1H, H-1), 5.30-5.20 (m, 2H, $-\mathrm{CH}_{2}-$ $\left.\mathrm{CH}=\mathrm{CH}_{2}\right), 5.30(\mathrm{~s}, 1 \mathrm{H}, \mathrm{H}-1), 5.18(\mathrm{~s}, 1 \mathrm{H}, \mathrm{H}-1), 5.12(\mathrm{~s}$, 1H, H-1), 5.11 (s, 1H, H-1), 4.66-3.92 (m, 26H, 4H-2, $\left.2 \mathrm{H}-3, \quad 6 \mathrm{H}-5, \quad 12 \mathrm{H}-6, \quad-\mathrm{CH}_{2}-\mathrm{CH}=\mathrm{CH}_{2}\right) ;{ }^{13} \mathrm{C} \quad \mathrm{NMR}$ $\left(100 \mathrm{MHz}, \mathrm{CDCl}_{3}\right): \delta 167.14,166.75,166.63,166.16$, $166.14,166.10,166.02,165.97,165.77,165.73,165.63$, $165.56,165.52,165.37,165.29,165.09,164.78,100.28$, $100.26,99.92,99.74,98.81,98.04,72.96,71.33,71.20$, $70.14,70.04,69.73,69.67,69.57,69.20,69.08,68.78$, $68.02,66.94,66.37,65.29,63.78,63.66,63.48,63.33$, 62.99, 62.16, 60.43. Anal. Calcd for $\mathrm{C}_{158} \mathrm{H}_{134} \mathrm{O}_{48}$ : C, 67.76; H, 4.82. Found: C, 67.93; H, 4.91.

4.12. Allyl 2,3,4,6-tetra- $O$-benzoyl- $\alpha$-D-mannopyranosyl$(1 \rightarrow 2)-3,4,6$-tri- $O$-benzoyl- $\alpha$-D-mannopyranosyl$(1 \rightarrow 6)$-[2,3,5,6-tetra- $O$-benzoyl- $\beta$-D-galactofuranosyl$(1 \rightarrow 3)$ ]-2,4-di- $O$-benzoyl- $\alpha$-D-mannopyranosyl-( $1 \rightarrow 2)$ $[2,3,5,6$-tetra- $O$-benzoyl- $\beta$-D-galactofuranosyl- $(1 \rightarrow 3)]-$ 4,6-di- $O$-benzoyl- $\alpha$-D-mannopyranosyl- $(1 \rightarrow 2)-3,4,6$-tri$O$-benzoyl- $\alpha$-D-mannopyranosyl-( $\rightarrow$ 2)-3,4,6-tri- $O$-benzoyl- $\alpha$-D-mannopyranoside 17

Acceptor $15(0.29 \mathrm{~g}, 0.10 \mathrm{mmol})$ was coupled with donor $16(0.18 \mathrm{~g}, 0.24 \mathrm{mmol})$ as described in the general procedure, and the product purified by chromatography with 1.5:1 petroleum ether-EtOAc as the eluent to give $17(0.30 \mathrm{~g}, 73 \%)$ as a foamy solid. $[\alpha]_{\mathrm{D}}^{25}=-18.6(c 1.0$, $\left.\mathrm{CHCl}_{3}\right) ;{ }^{1} \mathrm{H} \mathrm{NMR}\left(400 \mathrm{MHz}, \mathrm{CDCl}_{3}\right): \delta 8.14-6.89(\mathrm{~m}$, $125 \mathrm{H}, 25 P h), 6.32\left(\mathrm{dd}, 1 \mathrm{H}, J_{3,4}=J_{4,5}=10.0 \mathrm{~Hz}, \mathrm{H}-4\right)$, 
6.14-5.75 (m, 14H, Galf 2H-5, Manp 2H-2, 4H-3, 5H-4, $\left.-\mathrm{CH}_{2}-\mathrm{CH}=\mathrm{CH}_{2}\right), 5.57\left(\mathrm{~d}, 1 \mathrm{H}, J_{3,4}=4.5 \mathrm{~Hz}\right.$, Galf $\left.\mathrm{H}-3\right)$, $5.49\left(\mathrm{~d}, 1 \mathrm{H}, J_{3,4}=5.1 \mathrm{~Hz}\right.$, Galf $\left.\mathrm{H}-3\right), 5.48$ (s, $2 \mathrm{H}$, Gal $f$ 2H-1), 5.44 (s, 1H, Manp H-1), 5.41 (s, 2H, Galf 2H-2), 5.33 (s, 1H, Manp H-1), 5.29 (s, 2H, Manp 2H-1), 5.27$5.13\left(\mathrm{~m}, 2 \mathrm{H},-\mathrm{CH}_{2}-\mathrm{CH}=\mathrm{CH}_{2}\right), 4.95$ (s, $1 \mathrm{H}$, Man $\left.p \mathrm{H}-1\right)$, 4.75-3.86 (m, 32H, Galf 2H-4, 4H-6, Manp 4H-2, 2H-3, $\left.6 \mathrm{H}-5,12 \mathrm{H}-6,-\mathrm{CH}_{2}-\mathrm{CH}=\mathrm{CH}_{2}\right) ;{ }^{13} \mathrm{C} \mathrm{NMR}(100 \mathrm{MHz}$, $\left.\mathrm{CDCl}_{3}\right): \delta 167.07,166.09,165.95,165.82,165.69,165.65$, $165.57,165.55,165.52,165.41,165.33,165.28,165.26$, $165.01,164.99,164.84,164.77,104.27,102.24,100.65$, $100.56,100.17,99.74,98.96,97.98,83.06,82.83$, $82.22,81.62,75.10,73.91,71.91,71.59,70.92,70.20$, $70.07,69.94,69.77,69.66,69.54,68.99,68.86$, $68.73,68.64,67.88,67.52,67.25,66.94,66.63,66.30$, $65.74,63.93,63.71,63.35,62.08,60.34$. Anal. Calcd for $\mathrm{C}_{226} \mathrm{H}_{186} \mathrm{O}_{66}: \mathrm{C}, 68.58 ; \mathrm{H}, 4.74$. Found: C, 68.75; H, 4.78.

\subsection{2,3,4,6-Tetra- $O$-benzoyl- $\alpha$-D-mannopyranosyl- $(1 \rightarrow 2)-3,4,6$-tri- $O$-benzoyl- $\alpha$-D-mannopyranosyl- $(1 \rightarrow 6)-[2,3,5,6$-tetra- $O$-benzoyl- $\beta$-D-galactofuranosyl- $(1 \rightarrow 3)]-2,4-d i-O$-benzoyl- $\alpha$-D-mannopyranosyl-( $\rightarrow 2)$ - $[2,3,5,6$-tetra- $O$-benzoyl- $\beta$-D-galactofuranosyl- $(1 \rightarrow 3)]-$ 4,6-di- $O$-benzoyl- $\alpha$-D-mannopyranosyl-( $1 \rightarrow 2)-3,4,6$-tri- $O$-benzoyl- $\alpha$-D-mannopyranosyl-( $\rightarrow 2)$-3,4,6-tri- $O$-benz- oyl- $\alpha$-D-mannopyranosyl trichloroacetimidate 19}

Deallylation of tetrasaccharide $17(0.28 \mathrm{~g}, 71 \mu \mathrm{mol})$ followed by trichloroacetimidation under the same conditions as those used for the preparation of 7 from 5 gave a residue, which was purified by flash chromatography (3:2 petroleum ether-EtOAc) to give $19(0.20 \mathrm{~g}, 71 \%$ for two steps) as a foamy solid. $[\alpha]_{\mathrm{D}}^{25}=-13.5$ (c 1.0, $\left.\mathrm{CHCl}_{3}\right) ;{ }^{1} \mathrm{H} \mathrm{NMR}\left(400 \mathrm{MHz}, \mathrm{CDCl}_{3}\right): \delta 8.63(\mathrm{~s}, 1 \mathrm{H}$, $\left.\mathrm{CNHCCl}_{3}\right), 8.14-6.92(\mathrm{~m}, 125 \mathrm{H}, 25 \mathrm{Ph}), 6.62(\mathrm{~s}, 1 \mathrm{H}$, $\operatorname{Man} p \mathrm{H}-1), 6.31\left(\mathrm{dd}, 1 \mathrm{H}, J_{3,4}=J_{4,5}=10.4 \mathrm{~Hz}, \mathrm{H}-4\right)$, 6.15-5.74 (m, 13H, Galf 2H-5, Manp 2H-2, 4H-3, 5H-4), $5.60(\mathrm{~s}, 1 \mathrm{H}, \operatorname{Man} p \mathrm{H}-1), 5.57\left(\mathrm{~d}, 1 \mathrm{H}, J_{3,4}=4.5 \mathrm{~Hz}\right.$, Gal $f$ $\mathrm{H}-3), 5.50$ (d, $\left.1 \mathrm{H}, J_{3,4}=5.1 \mathrm{~Hz}, \mathrm{Gal} f \mathrm{H}-3\right), 5.47$ (s, 2H, Galf 2H-1), 5.42 (s, 2H, Galf 2H-2), 5.33 (s, 2H, Man $p$ 2H-1), 5.30 (s, 1H, Manp H-1), 4.92 (s, 1H, Manp H-1), 4.78-3.92 (m, 30H, Galf 2H-4, 4H-6, Manp 4H-2, 2H-3, $6 \mathrm{H}-5$, 12H-6). Anal. Calcd for $\mathrm{C}_{225} \mathrm{H}_{182} \mathrm{Cl}_{3} \mathrm{NO}_{66}$ : C, 66.53; H, 4.52. Found: C, 66.25; H, 4.44.

\subsection{Allyl 2,3,5,6-tetra- $O$-benzoyl- $\beta$-D-galactofuranosyl- $(1 \rightarrow 3)-4,6$-di- $O$-benzylidene- $\alpha$-D-mannopyranoside 21}

As described in the general procedure, $16(0.36 \mathrm{~g}$, $0.49 \mathrm{mmol})$ and $20(0.18 \mathrm{~g}, 0.59 \mathrm{mmol})$ were coupled, and the product purified by silica-gel column chromatography with 2.5:1 petroleum ether-EtOAc as the eluent to give $7(0.43 \mathrm{~g}, 71 \%)$ as a foamy solid. $[\alpha]_{\mathrm{D}}^{25}=+10.2$ (c 1.0, $\left.\mathrm{CHCl}_{3}\right) ;{ }^{1} \mathrm{H}$ NMR $\left(400 \mathrm{MHz}, \mathrm{CDCl}_{3}\right): \delta 8.04$ 7.19 (m, 25H, 5Ph), 5.96-5.86 (m, 2H, Galf $\mathrm{H}-5,-\mathrm{CH}_{2}-$ $\left.\mathrm{CH}=\mathrm{CH}_{2}\right), 5.55\left(\mathrm{dd}, 1 \mathrm{H}, J_{2,3}=1.2 \mathrm{~Hz}, J_{3,4}=5.5 \mathrm{~Hz}\right.$, Galf $\mathrm{H}-3), 5.50$ (s, 1H, PhCH), 5.44 (s, 1H, Galf H-1), $5.43\left(\mathrm{~d}, 1 \mathrm{H}, J_{2,3}=1.2 \mathrm{~Hz}\right.$, Gal $\left.f \mathrm{H}-2\right), 5.34-5.22(\mathrm{~m}, 2 \mathrm{H}$, $\left.-\mathrm{CH}_{2}-\mathrm{CH}=\mathrm{CH}_{2}\right), 4.99\left(\mathrm{~d}, 1 \mathrm{H}, J_{1,2}=1.2 \mathrm{~Hz}, \operatorname{Man} p\right.$
$\mathrm{H}-1), 4.74\left(\mathrm{dd}, 1 \mathrm{H}, J_{3,4}=5.5 \mathrm{~Hz}, J_{2,3}=3.1 \mathrm{~Hz}\right.$, Gal $f \mathrm{H}-$ 4), 4.60-3.82 (m, 10H, Galf H-6, Manp H-2, H-3, H-4, $\mathrm{H}-5, \mathrm{H}-6,-\mathrm{CH}_{2}-\mathrm{CH}=\mathrm{CH}_{2}$ ). Anal. Calcd for $\mathrm{C}_{50} \mathrm{H}_{46} \mathrm{O}_{15}$ : C, 67.71; H, 5.23. Found: C, 67.97; H, 5.30.

\subsection{Allyl 2,3,5,6-tetra- $O$-benzoyl- $\beta$-D-galactofuranosyl- $(1 \rightarrow 3)-2-O$-acetyl-4,6-di- $O$-benzylidene- $\alpha$-D-mannopyr- anoside 22}

To a solution of compound $21(0.42 \mathrm{~g}, 0.47 \mathrm{mmol})$ in pyridine $(10 \mathrm{~mL})$ was added $\mathrm{Ac}_{2} \mathrm{O}(5 \mathrm{~mL}, 5 \mathrm{mmol})$. The reaction mixture was stirred at $\mathrm{rt}$ for $12 \mathrm{~h}$ and concentrated to give the crude product, which was purified by flash chromatography (2.5:1 petroleum ether-EtOAc) to give $22(0.42 \mathrm{~g}, 96 \%)$ as a foamy solid. $[\alpha]_{\mathrm{D}}^{25}=-14.5(c$ $\left.1.3, \mathrm{H}_{2} \mathrm{O}\right) ;{ }^{1} \mathrm{H} \mathrm{NMR}\left(400 \mathrm{MHz}, \mathrm{CDCl}_{3}\right): \delta 8.02-7.16(\mathrm{~m}$, $25 \mathrm{H}, 5 \mathrm{Ph}), 5.95-5.85$ (m, 2H, Galf $\mathrm{H}-5,-\mathrm{CH}_{2}-$ $\left.\mathrm{CH}=\mathrm{CH}_{2}\right), \quad 5.53 \quad(\mathrm{~s}, \quad 1 \mathrm{H}, \mathrm{PhCH}), 5.47 \quad(\mathrm{~d}, 1 \mathrm{H}$, $J_{3,4}=5.4 \mathrm{~Hz}$, Gal $\left.f \mathrm{H}-3\right), 5.43$ (s, $\left.1 \mathrm{H}, \mathrm{Gal} f \mathrm{H}-1\right), 5.42$ (dd, $\left.1 \mathrm{H}, J_{1,2}=1.4 \mathrm{~Hz}, J_{2,3}=3.5 \mathrm{~Hz}, \operatorname{Man} p \mathrm{H}-2\right), 5.41(\mathrm{~s}, 1 \mathrm{H}$, Galf $\mathrm{H}-2), 5.33-5.22\left(\mathrm{~m}, 2 \mathrm{H},-\mathrm{CH}_{2}-\mathrm{CH}=\mathrm{CH}_{2}\right), 4.86(\mathrm{~d}$, $\left.1 \mathrm{H}, \quad J_{1,2}=1.3 \mathrm{~Hz}, \quad \operatorname{Man} p \mathrm{H}-1\right), 4.70 \quad(\mathrm{dd}, \quad 1 \mathrm{H}$, $J_{3,4}=5.3 \mathrm{~Hz}, J_{2,3}=3.0 \mathrm{~Hz}$, Galf $\left.\mathrm{H}-4\right), 4.64-3.81(\mathrm{~m}, 9 \mathrm{H}$, Galf H-6, Man $p$ H-3, H-4, H-5, H-6, $-\mathrm{CH}_{2}-\mathrm{CH}=\mathrm{CH}_{2}$ ). Anal. Calcd for $\mathrm{C}_{52} \mathrm{H}_{48} \mathrm{O}_{16}$ : C, 67.23; H, 5.21. Found: C, 67.01; H, 5.15 .

4.16. Allyl 2,3,5,6-tetra- $O$-benzoyl- $\beta$-D-galactofuranosyl$(1 \rightarrow 3)-2-O$-acetyl-4,6-di- $O$-benzoyl- $\alpha$-D-mannopyranoside 24

Compound $22(0.40 \mathrm{~g}, 0.43 \mathrm{mmol})$ was dissolved in $90 \%$ TFA $(10 \mathrm{~mL})$, and the mixture stirred for $2 \mathrm{~h}$ at $\mathrm{rt}$, at the end of which time TLC (2:1 petroleum ether-EtOAc) indicated that the reaction was complete. The mixture was diluted with toluene $(40 \mathrm{~mL})$ and concentrated in vacuo directly to give crude product 23. Benzoylation of compound $\mathbf{2 3}$ under the same conditions used in the preparation of $\mathbf{5}$ from $\mathbf{4}$ gave a residue, which was purified by flash chromatography (3:1 petroleum etherEtOAc) to give $\mathbf{2 4}(0.31 \mathrm{~g}, 72 \%$ for two steps $)$ as a foamy solid. $[\alpha]_{\mathrm{D}}^{25}=+8.7$ (c $\left.1.0, \mathrm{CHCl}_{3}\right) ;{ }^{1} \mathrm{H} \mathrm{NMR}(400 \mathrm{MHz}$, $\left.\mathrm{CDCl}_{3}\right): \delta 8.03-7.15(\mathrm{~m}, 30 \mathrm{H}, 6 \mathrm{Ph}), 5.98-5.86(\mathrm{~m}, 2 \mathrm{H}$, Galf $\left.\mathrm{H}-5, \quad-\mathrm{CH}_{2}-\mathrm{CH}=\mathrm{CH}_{2}\right), \quad 5.72 \quad(\mathrm{dd}, \quad 1 \mathrm{H}$, $\left.J_{3,4}=J_{4,5}=10.0 \mathrm{~Hz}, \operatorname{Man} p \mathrm{H}-4\right), 5.49$ (s, 1H, Gal $\left.f \mathrm{H}-1\right)$, $5.47\left(\mathrm{dd}, 1 \mathrm{H}, J_{1,2}=1.7 \mathrm{~Hz}, J_{2.3}=3.4 \mathrm{~Hz}, \operatorname{Man} p \mathrm{H}-2\right)$, $5.43\left(\mathrm{~d}, 1 \mathrm{H}, J_{3,4}=5.1 \mathrm{~Hz}, \mathrm{Gal} f \mathrm{H}-3\right), 5.35$ (s, $1 \mathrm{H}$, Galf $\mathrm{H}-2), 5.33-5.22\left(\mathrm{~m}, 2 \mathrm{H},-\mathrm{CH}_{2}-\mathrm{CH}=\mathrm{CH}_{2}\right), 4.98(\mathrm{~d}, 1 \mathrm{H}$, $\left.J_{1,2}=1.7 \mathrm{~Hz}, \operatorname{Man} p \mathrm{H}-1\right), 4.70\left(\mathrm{dd}, 1 \mathrm{H}, J_{3,4}=5.3 \mathrm{~Hz}\right.$, $J_{2,3}=3.0 \mathrm{~Hz}$, Gal $\left.f \mathrm{H}-4\right), 4.62-4.05$ (m, 9H, Galf $\mathrm{H}-4, \mathrm{H}-$ 6, Man $p$ H-3, H-5, H-6, $-\mathrm{CH}_{2}-\mathrm{CH}=\mathrm{CH}_{2}$ ). Anal. Calcd for $\mathrm{C}_{59} \mathrm{H}_{52} \mathrm{O}_{18}$ : C, 67.55; H, 5.00. Found: C, 67.63; H, 4.95 .

4.17. Allyl 2,3,5,6-tetra- $O$-benzoyl- $\beta$-D-galactofuranosyl$(1 \rightarrow 3)-4,6$-di- $O$-benzoyl- $\alpha$-D-mannopyranoside 25

Deacetylation of $24(0.29 \mathrm{~g}, 0.27 \mathrm{mmol})$ under the same conditions as those used in the preparation of $\mathbf{1 5}$ from 14 gave the crude product, which was purified by flash 
chromatography $(2.5: 1$ petroleum ether-EtOAc) to furnish $25(0.21 \mathrm{~g}, 75 \%)$ as a foamy solid. $[\alpha]_{\mathrm{D}}=+17.2$ (c 1.0, $\left.\mathrm{CHCl}_{3}\right) ;{ }^{1} \mathrm{H}$ NMR $\left(400 \mathrm{MHz}, \mathrm{CDCl}_{3}\right): \delta 8.00$ $7.23(\mathrm{~m}, \quad 30 \mathrm{H}, 6 \mathrm{Ph}), \quad 5.99-5.89\left(\mathrm{~m}, \quad 1 \mathrm{H}, \quad-\mathrm{CH}_{2-}\right.$ $\left.\mathrm{CH}=\mathrm{CH}_{2}\right), 5.82-5.77(\mathrm{~m}, 2 \mathrm{H}, \mathrm{Gal} f \mathrm{H}-5$, Man $p \mathrm{H}-4)$, $5.58\left(\mathrm{dd}, 1 \mathrm{H}, J_{2,3}=1.5 \mathrm{~Hz}, J_{3.4}=5.1 \mathrm{~Hz}, \mathrm{Gal} f \mathrm{H}-3\right), 5.47$ $(\mathrm{s}, 1 \mathrm{H}, \mathrm{Gal} f \mathrm{H}-1), 5.36\left(\mathrm{~d}, 1 \mathrm{H}, J_{2,3}=1.5 \mathrm{~Hz}, \mathrm{Gal} f \mathrm{H}-2\right)$, 5.33-5.22 (m, 2H, $\left.-\mathrm{CH}_{2}-\mathrm{CH}=\mathrm{CH}_{2}\right), 5.10(\mathrm{~d}, 1 \mathrm{H}$, $\left.J_{1,2}=1.4 \mathrm{~Hz}, \operatorname{Man} p \mathrm{H}-1\right), 5.47\left(\mathrm{dd}, 1 \mathrm{H}, J_{1,2}=1.7 \mathrm{~Hz}\right.$, $\left.J_{2,3}=3.4 \mathrm{~Hz}, \operatorname{Man} p \mathrm{H}-2\right), 4.70\left(\mathrm{dd}, 1 \mathrm{H}, J_{3,4}=5.3 \mathrm{~Hz}\right.$, $\left.J_{2,3}=3.0 \mathrm{~Hz}, \mathrm{Gal} f \mathrm{H}-4\right), 4.57-4.12(\mathrm{~m}, 10 \mathrm{H}$, Gal $f \mathrm{H}-4$, $\mathrm{H}-6$, Manp H-2, H-3, H-5, H-6, $-\mathrm{CH}_{2}-\mathrm{CH}=\mathrm{CH}_{2}$ ). Anal. Calcd for $\mathrm{C}_{57} \mathrm{H}_{50} \mathrm{O}_{17}: \mathrm{C}, 67.99 ; \mathrm{H}, 5.00$. Found: $\mathrm{C}$, 68.12; H, 5.09.

\subsection{Allyl 6- $O$-acetyl-2,3,4-tri- $O$-benzoyl- $\alpha$-D-manno- pyranosyl-[2,3,5,6-tetra- $O$-benzoyl- $\beta$-D-galactofuranosyl- $(1 \rightarrow 3)$ ]-4,6-di- $O$-benzoyl- $\alpha$-D-mannopyranoside 27}

Donor $26(0.15 \mathrm{~g}, 0.22 \mathrm{mmol})$ was coupled with acceptor $25(0.18 \mathrm{~g}, 0.18 \mathrm{mmol})$ as described in the general procedure, and the product purified by chromatography with 2:1 petroleum ether-EtOAc as the eluent to give $\mathbf{2 7}$ $(0.22 \mathrm{~g}, 78 \%)$ as a foamy solid. $[\alpha]_{\mathrm{D}}^{25}=-20.2(\mathrm{c} 1.0$, $\left.\mathrm{CHCl}_{3}\right) ;{ }^{1} \mathrm{H}$ NMR $\left(400 \mathrm{MHz}, \mathrm{CDCl}_{3}\right): \delta 8.09-6.98(\mathrm{~m}$, $45 \mathrm{H}, 9 P h), 6.07-5.88$ (m, 6H, Gal $f \mathrm{H}-5$, Man $p$ H-2', H$\left.3^{\prime}, \quad \mathrm{H}-4, \quad \mathrm{H}-4^{\prime}, \quad-\mathrm{CH}_{2}-\mathrm{CH}=\mathrm{CH}_{2}\right), \quad 5.62(\mathrm{~d}, \quad 1 \mathrm{H}$, $\left.J_{1^{\prime}, 2^{\prime}}=1.0 \mathrm{~Hz}, \operatorname{Man} p \mathrm{H}-1^{\prime}\right), 5.52\left(\mathrm{~d}, 1 \mathrm{H}, J_{3,4}=4.4 \mathrm{~Hz}\right.$, Gal $f \mathrm{H}-3), 5.51$ (s, $1 \mathrm{H}, \mathrm{Gal} f \mathrm{H}-1), 5.43$ (s, $1 \mathrm{H}, \mathrm{Gal} f \mathrm{H}-2)$, $5.36-5.24\left(\mathrm{~m}, 2 \mathrm{H},-\mathrm{CH}_{2}-\mathrm{CH}=\mathrm{CH}_{2}\right), 5.18(\mathrm{~d}, 1 \mathrm{H}$, $\left.J_{1,2}=1.2 \mathrm{~Hz}, \operatorname{Man} p \mathrm{H}-1\right), 4.65-4.10(\mathrm{~m}, 13 \mathrm{H}, \mathrm{Gal} f \mathrm{H}-4$, H-6, Manp H-2, H-3, H-5, H-5', H-6, H-6', $-\mathrm{CH}_{2}-$ $\left.\mathrm{CH}=\mathrm{CH}_{2}\right) ;{ }^{13} \mathrm{C}$ NMR $\left(100 \mathrm{MHz}, \mathrm{CDCl}_{3}\right): \delta 170.49$, $166.32,166.12$, 165.62, 165.55, 165.11, 164.98, 164.63, $102.83,100.70,98.19,82.82,75.75,72.73,70.06,69.74$, 69.26, 69.05, 68.69, 67.64, 67.30, 64.06, 63.82, 63.07, 20.67. Anal. Calcd for $\mathrm{C}_{86} \mathrm{H}_{74} \mathrm{O}_{26}: \mathrm{C}, 67.80 ; \mathrm{H}, 4.90$. Found: C, 68.03; H, 5.01 .

4.19. Allyl 2,3,4-tri- $O$-benzoyl- $\alpha$-D-mannopyranosyl$(1 \rightarrow 2)$-[2,3,5,6-tetra- $O$-benzoyl- $\beta$-D-galactofuranosyl$(1 \rightarrow 3)$ ]-4,6-di- $O$-benzoyl- $\alpha$-D-mannopyranoside 28

Deacetylation of compound $27(0.20 \mathrm{~g}, 0.13 \mathrm{mmol})$ was carried out under the same conditions as those used in the preparation of $\mathbf{1 5}$ from $\mathbf{1 4}$, giving the crude product, which was purified by flash chromatography (2:1 petroleum ether-EtOAc) to give $28(0.16 \mathrm{~g}, 81 \%)$ as a foamy solid. $[\alpha]_{\mathrm{D}}^{25}=-52.5\left(\right.$ c $\left.1.0, \mathrm{CHCl}_{3}\right) ;{ }^{1} \mathrm{H}$ NMR $\left(400 \mathrm{MHz}, \mathrm{CDCl}_{3}\right): \delta 7.98-6.99(\mathrm{~m}, 45 \mathrm{H}, 9 P h), 6.12(\mathrm{dd}$, $\left.1 \mathrm{H}, J_{2^{\prime}, 3^{\prime}}=3.4 \mathrm{~Hz}, J_{3^{\prime}, 4^{\prime}}=10.0 \mathrm{~Hz}, \operatorname{Man} p \mathrm{H}-3^{\prime}\right) 6.05(\mathrm{dd}$, $\left.1 \mathrm{H}, J_{1^{\prime}, 2^{\prime}}=1.5 \mathrm{~Hz}, J_{2^{\prime}, 3^{\prime}}=3.4 \mathrm{~Hz}, \operatorname{Man} p \mathrm{H}-2^{\prime}\right), 5.98-5.87$ (m, 3H, Galf $\mathrm{H}-5$, Man $p \mathrm{H}-4^{\prime},-\mathrm{CH}_{2}-\mathrm{CH}=\mathrm{CH}_{2}$ ), 5.84 $\left(\mathrm{dd}, 1 \mathrm{H}, J_{3,4}=J_{4,5}=10.0 \mathrm{~Hz}, \operatorname{Man} p \mathrm{H}-4\right), 5.67(\mathrm{~d}, 1 \mathrm{H}$, $\left.J_{1^{\prime}, 2^{\prime}}=1.5 \mathrm{~Hz}, \operatorname{Man} p \mathrm{H}-1^{\prime}\right), 5.52\left(\mathrm{~d}, 1 \mathrm{H}, J_{3,4}=4.9 \mathrm{~Hz}\right.$, Gal $f \mathrm{H}-3), 5.51(\mathrm{~s}, 1 \mathrm{H}, \mathrm{Gal} f \mathrm{H}-1), 5.42(\mathrm{~s}, 1 \mathrm{H}, \mathrm{Gal} f \mathrm{H}-2)$, 5.34-5.23 (m, $\left.2 \mathrm{H},-\mathrm{CH}_{2}-\mathrm{CH}=\mathrm{CH}_{2}\right), 5.16(\mathrm{~d}, 1 \mathrm{H}$, $J_{1,2}=1.6 \mathrm{~Hz}$, Man $\left.p \mathrm{H}-1\right), 4.64-3.80(\mathrm{~m}, 13 \mathrm{H}$, Gal $f \mathrm{H}-4$, H-6, Manp H-2, H-3, H-5, H-5', H-6, H-6', $-\mathrm{CH}_{2}-$ $\mathrm{CH}=\mathrm{CH}_{2}$ ). Anal. Calcd for $\mathrm{C}_{84} \mathrm{H}_{72} \mathrm{O}_{25}$ : C, 68.10; H, 4.90. Found: C, 68.21; H, 4.95.
4.20. Allyl 2,3,4,6-tetra- $O$-benzoyl- $\alpha$-D-mannopyranosyl$(1 \rightarrow 2)$-3,4,6-tri- $O$-benzoyl- $\alpha$-D-mannopyranosyl$(1 \rightarrow 6)-[2,3,5,6$-tetra- $O$-benzoyl- $\beta$-D-galactofuranosyl$(1 \rightarrow 3)]-2,4-d i-O$-benzoyl- $\alpha$-D-mannopyranosyl- $(1 \rightarrow 2)-$ $[2,3,5,6$-tetra- $O$-benzoyl- $\beta$-D-galactofuranosyl- $(1 \rightarrow 3)]-$ 4,6-di- $O$-benzoyl- $\alpha$-D-mannopyranosyl- $(1 \rightarrow 2)-3,4,6$-tri$O$-benzoyl- $\alpha$-D-mannopyranosyl-( $1 \rightarrow 2)-3,4,6$-tri- $O$-benzoyl- $\alpha$-D-mannopyranosyl-( $(1 \rightarrow 6)-2,3,4$-tri- $O$-benzoyl- $\alpha-$ D-mannopyranosyl-( $1 \rightarrow 2)$-[2,3,5,6-tetra- $O$-benzoyl- $\beta$-Dgalactofuranosyl-( $(\rightarrow 3)$ ]-4,6-di- $O$-benzoyl- $\alpha-\mathrm{D}$-mannopyranoside 29

Donor $19(0.19 \mathrm{~g}, 46 \mu \mathrm{mol})$ was coupled with acceptor 28 $(82 \mathrm{mg}, 55 \mu \mathrm{mol})$ as described in the general procedure to give the crude product, which was purified by flash chromatography (1.2:1 petroleum ether-EtOAc) to give target compound $29(0.20 \mathrm{~g}, 79 \%)$ as a foamy solid. $[\alpha]_{\mathrm{D}}^{25}=-41.7\left(\mathrm{c} 1.0, \mathrm{CHCl}_{3}\right) ;{ }^{1} \mathrm{H}$ NMR $(400 \mathrm{MHz}$, $\left.\mathrm{CDCl}_{3}\right): \delta 8.04-6.91(\mathrm{~m}, 170 \mathrm{H}, 34 P h), 6.33(\mathrm{dd}, 1 \mathrm{H}$, $\left.J_{3,4}=J_{4,5}=9.9 \mathrm{~Hz}, \quad \mathrm{H}-4\right), \quad 6.21 \quad\left(\mathrm{dd}, \quad 1 \mathrm{H}, \quad J_{3,4}=\right.$ $\left.J_{4,5}=10.0 \mathrm{~Hz}, \mathrm{H}-4\right), 6.15-5.63(\mathrm{~m}, 18 \mathrm{H}, \mathrm{Gal} f 3 \mathrm{H}-5$, Manp $\left.3 \mathrm{H}-2,5 \mathrm{H}-3,6 \mathrm{H}-4,-\mathrm{CH}_{2}-\mathrm{CH}=\mathrm{CH}_{2}\right), 5.59(\mathrm{~d}, 1 \mathrm{H}$, $\left.J_{3.4}=4.4 \mathrm{~Hz}, \mathrm{Gal} f \mathrm{H}-3\right), 5.50-5.46(\mathrm{~m}, 5 \mathrm{H}$, Galf $3 \mathrm{H}-1$, $2 \mathrm{H}-3), 5.43$ (s, $2 \mathrm{H}$, Galf $2 \mathrm{H}-2), 5.34$ (s, $1 \mathrm{H}$, Manp H-1), 5.32 (s, $1 \mathrm{H}, \mathrm{Gal} f \mathrm{H}-2), 5.28$ (s, $1 \mathrm{H}, \operatorname{Man} p \mathrm{H}-1), 5.26$ (s, $2 \mathrm{H}$, Manp $2 \mathrm{H}-1), 5.24-5.07\left(\mathrm{~m}, 2 \mathrm{H},-\mathrm{CH}_{2}-\mathrm{CH}=\mathrm{CH}_{2}\right)$, 5.23 (s, 2H, Manp 2H-1), 5.21 (s, 1H, Manp H-1), 4.92 (s, 1H, Man $p \mathrm{H}-1$ ), 4.68-3.68 (m, 43H, Galf 3H-4, 6H-6, Man $\left.p 5 \mathrm{H}-2,3 \mathrm{H}-3,8 \mathrm{H}-5,16 \mathrm{H}-6,-\mathrm{CH}_{2}-\mathrm{CH}=\mathrm{CH}_{2}\right) ;{ }^{13} \mathrm{C}$ NMR $\left(100 \mathrm{MHz}, \mathrm{CDCl}_{3}\right): \delta 166.88,166.23,166.00$, $165.94,165.80,165.75,165.72,165.55,165.48,165.43$, $165.36,165.29,165.19,165.16,165.07,164.76,164.73$, $164.64,164.58,163.17,104.12,102.73,102.21,101.12$, $100.61,100.19,99.62,98.83,98.44,98.32,95.92,82.85$, $82.76,82.62,82.10,81.50,75.24,74.78,73.78,72.87$, $71.99,71.72,71.46,70.33,70.15,69.98,69.86,69.79$, $69.51,69.36,68.93,68.74,67.56,67.20,67.02,66.85$, $66.65,66.51,66.24,66.05,63.97,63.75,63.49,63.21$, 63.05, 61.83. Anal. Calcd for $\mathrm{C}_{307} \mathrm{H}_{252} \mathrm{O}_{90}$ : C, 68.52; $\mathrm{H}$, 4.72. Found: C, 68.78; H, 4.64.

4.21. Allyl $\alpha$-D-mannopyranosyl-( $1 \rightarrow 2)$ - $\alpha$-D-mannopyranosyl-( $1 \rightarrow 6)$-[ $[\beta$-D-galactofuranosyl- $(1 \rightarrow 3)]-\alpha-\mathrm{D}-$ mannopyranosyl-( $(1 \rightarrow 2)$-[ $\beta$-D-galactofuranosyl-( $1 \rightarrow 3)]$ $\alpha$-D-mannopyranosyl-(1 $\rightarrow 2)$ - $\alpha$-D-mannopyranosyl$(1 \rightarrow 2)-\alpha$-D-mannopyranosyl-( $1 \rightarrow 6)$ - $\alpha$-D-mannopyranosyl-(1 $\rightarrow 2)$-[ $\beta$-D-galactofuranosyl- $(1 \rightarrow 3)]-\alpha-D$-mannopyranoside 30

Undecasaccharide $29(0.19 \mathrm{~g}, 34 \mu \mathrm{mol})$ was dissolved in satd $\mathrm{NH}_{3}-\mathrm{MeOH}(30 \mathrm{~mL})$. After $96 \mathrm{~h}$ at $\mathrm{rt}$, the reaction mixture was concentrated, and the residue purified by chromatography on Sephadex LH-20 (MeOH) to afford $30(54 \mathrm{mg}, 85 \%)$ as a foamy solid. $[\alpha]_{\mathrm{D}}^{25}=+8.5(c 1.0$, $\left.\mathrm{H}_{2} \mathrm{O}\right) ;{ }^{1} \mathrm{H}$ NMR $\left(400 \mathrm{MHz}, \mathrm{D}_{2} \mathrm{O}\right): \delta$ 5.88-5.98 (m, $\left.1 \mathrm{H},-\mathrm{CH}_{2}-\mathrm{CH}=\mathrm{CH}_{2}\right), 5.35-5.31(\mathrm{dd}, 1 \mathrm{H}, J=17.1 \mathrm{~Hz}$, $\left.-\mathrm{CH}_{2}-\mathrm{CH}=\mathrm{CH}_{\text {trans }}\right), 5.26-5.24(\mathrm{dd}, 1 \mathrm{H}, J=10.4 \mathrm{~Hz}$, $\left.-\mathrm{CH}_{2}-\mathrm{CH}=\mathrm{CH}_{\text {cis }}\right), 5.20,5.16,5.15,5.11,5.07,5.02,4.99$ $(7 \mathrm{~s}, 11 \mathrm{H}, 11 \mathrm{H}-1) ;{ }^{13} \mathrm{C}$ NMR $\left(100 \mathrm{MHz}, \mathrm{D}_{2} \mathrm{O}\right): \delta 104.88$, $104.85,104.35,102.28,101.71,101.61,100.76,100.69$, $98.26,98.10,97.59,78.79,78.68,78.47,77.05,76.84$, $75.78,75.55,75.23,74.24,73.99,73.34,73.26,73.21$, 
$72.95,72.68,71.80,71.58,70.72,70.69,70.64,70.46$, $70.32,70.26,70.03,69.97,69.91,68.15,67.09,67.03$, $66.89,66.50,65.97,65.40,65.29,65.23,64.51,62.84$, $62.78,61.17,60.94,60.88,60.82$. Anal. Calcd for $\mathrm{C}_{69} \mathrm{H}_{116} \mathrm{O}_{56}: \mathrm{C}, 45.00 ; \mathrm{H}, 6.35$. Found: $\mathrm{C}, 45.11 ; \mathrm{H}, 6.38$.

\section{Acknowledgements}

This work was supported by The Chinese Academy of Sciences (KZCX3-J-08) and by The National Natural Science Foundation of China (Projects 30070185 and 39970864).

\section{References and notes}

1. Hay, R. J. Br. J. Dermatol. 1982, 106, 1-7.

2. Dahl, M. V. J. Am. Acad. Dermatol. 1994, 31, S34-S41.

3. (a) Holden, A. J.; Hay, R. J.; MacDonald, D. M. J. Invest. Dermatol. 1981, 61, 207-211; (b) Blake, J. S.; Herron, M. J.; Nelson, R. D. J. Invest. Dermatol. 1991, 96, 657-661; (c) Grando, S. A.; Hostager, B. S.; Herron, M. J.; Dahl, M. V.; Nelson, R. D. J. Invest. Dermatol. 1992, 98, 876880 .

4. Ikuda, K.; Shibata, N.; Blake, J. S.; Dahl, M. V.; Nelson, R. D.; Hisamichi, K.; Kobayashi, H.; Suzuki, S.; Okawa, Y. Biochem. J. 1997, 323, 297-305.

5. Randell, K. D.; Johnston, B. D.; Brown, P. N.; Pinto, B. M. Carbohydr. Res. 2000, 325, 253-262.

6. (a) Zhang, J.; Kong, F. Tetrahedron: Asymmetry 2002, 13, 243-252; (b) Kong, F. Curr. Org. Chem. 2003, 7, 841-865.

7. (a) Ma, Z.; Zhang, J.; Kong, F. Tetrahedron: Asymmetry 2003, 14, 2595-2603; (b) Zhang, J.; Ma, Z.; Kong, F. Carbohydr. Res. 2003, 338, 2039-2046; (c) Zhang, J.; Kong, F. Bioorg. Med. Chem. 2003, 11, 4027-4037.

8. Zhang, J.; Ma, Z.; Kong, F. Carbohydr. Res. 2003, 338, $1711-1718$.

9. Zhu, Y.; Kong, F. Synlett 2000, 1783-1787.

10. Ogawa, T.; Yamamoto, H. Carbohydr. Res. 1985, 137, 7986.

11. Schmidt, R. R.; Kinzy, W. Adv. Carbohydr. Chem. Biochem. 1994, 50, 21-125.

12. (a) Byramova, N. E.; Ovchinnikov, M. V.; Backinowsky, L. V. Kochetkov. Carbohydr. Res. 1983, 124, c8; (b) Zhu, Y.; Kong, F. Chin. J. Chem. 2001, 19, 119-123.

13. Wang, H.; Zhang, G.; Ning, J. Carbohydr. Res. 2003, 338, $1033-1037$. 